\title{
HARMONIC MAPS AND CURVATURE COMPUTATIONS IN TEICHMÜLLER THEORY
}

\author{
Jürgen Jost
}

\section{Introduction}

The purpose of this paper is to display harmonic maps as a computational tool in Teichmüller theory. Let $\Sigma$ be a compact surface without boundary of genus $p \geq 2$, and let $g$ and $\gamma$ be (marked) conformal structures on $\Sigma$. Thus $(\Sigma, g)$ and $(\Sigma, \gamma)$ define elements of Teichmüller space $T_{p}$. Each such element carries a unique hyperbolic metric, and in the sequel it will always be equipped with this metric. It then follows from results of Al'ber, Eells-Sampson, Hartman, Schoen-Yau, and Sampson that there exists a unique harmonic map $u=u(z)$ from $(\Sigma, g)$ to $(\Sigma, \gamma)$ homotopic to the identity of $\Sigma$, and $u$ is a diffeomorphism. Furthermore, if $\varrho^{2}(u)$ is the image metric, then

$$
\Phi:=\varrho^{2}(u) u_{z} \bar{u}_{z} d z^{2}
$$

is a holomorphic quadratic differential on $(\Sigma, g)$, and this observation furnishes the link between harmonic maps and Teichmüller theory.

In order to explore this relation, one has to investigate the effect of variations of the domain structure $g$ and the image structure $\gamma$ on $\Phi$. Variations of the domain structures were first studied by Tromba [T2]. He in particular showed that the second variation of the energy of the harmonic map $u=u(g, \gamma)$ w.r.t. $g$ at $g=\gamma$ yields the Weil-Petersson metric on $T_{p}$. Wolf [Wf] then undertook a systematic investigation of variations of the image structure, and established important formulae for the effect of variations of $\gamma$ on $u$. Incidentally, the second variation of energy of $u(g, \gamma)$ w.r.t. at $g=\gamma$ again yields the Weil-Petersson metric, a result which can also be derived from Tromba's aforementioned result because the energy of $u(g, \gamma)$ is constant, i.e. independent of $g$. It was then possible to develop Teichmüller theory systematically in terms of harmonic maps, as carried out by Wolf [Wf] and the present author (Chapter 6 in [J2]).

The Weil-Petersson metric was introduced by Weil, and it was established by Weil and Ahlfors [A1] that it is a Kähler metric. Ahlfors [A2] also started to investigate its curvature properties and found that the Ricci and the holomorphic sectional curvature is negative. More recently, Tromba [T1] showed that the sectional curvature is negative. Different proofs of this result were given by Wolpert [WP] (in [Wp], such results are also independently attributed to Royden) and $\mathrm{Siu}[\mathrm{Si}]$. 
Here, we shall use harmonic maps to establish the curvature formula of the preceding authors for the Weil-Petersson metric. While this is not a new result, we believe that the computational technique introduced here will be important for further computations in Teichmüller theory. As an example of its potential, we point out that on the way at certain points we get more precise information than obtained in [Wp].

In [Wp], Wolpert also studied the geometry of the universal Teichmüller curve $\mathcal{T}_{p}$, with fibre over $g$ being the marked conformal surface determined by $g$. He showed that the first Chern class $c_{1}(L)$ of the vertical line bundle $L$ coming from the projection $\pi: \mathcal{T}_{p} \rightarrow T_{p}$ is negative. On the basis of this result, he suggested to use $-c_{1}(L)$ as a Kähler metric on $\mathcal{T}_{p}$. We shall use harmonic maps to study this metric. Wolpert showed that integrating the square of $-c_{1}(L)$ over the fibers of $\mathcal{T}_{p}$ yields the Weil-Petersson metric on $\mathcal{T}_{p}$. Nevertheless, contrary to what one might expect, it turns out that this metric is not negatively curved. Namely, the holomorphic sectional curvature in the fibre direction fails to be negative, although the intrinsic curvature of each fibre is -1 . Of course, this is in accord with the general principle in complex geometry that the intrinsic curvature of a submanifold is smaller than the curvature of the ambient manifold in the direction of this submanifold, the difference being given by the second fundamental form. On the basis of this result, there seems to be no point in presenting further computations for the curvature tensor of this metric, although we have carried out such computations. Anyway, one can obtain a negatively curved metric on $\mathcal{T}_{p}$ by a different method. Namely, there is a natural identification between $\mathcal{T}_{p}$ and the Teichmüller space $T_{p, 1}$ of surfaces of genus $p$ with one distinguished point, by just identifying the distinguished point on $(\Sigma, g)$ in $T_{p, 1}$ with the corresponding point in the fibre over $(\Sigma, g)$ in $\mathcal{T}_{p}$. In the same way as for $T_{p}$, one can define the Weil-Petersson metric for $T_{p, n}$ (Teichmüller space of surfaces with $n$ punctures), and since for example the computations in the present paper are purely formal, they also show the negativity of the sectional curvature of this metric. However, this metric on $T_{p, 1}$ does not reflect the fiber space structure of $\mathcal{T}_{p}$, and this was our reason for studying the above metric on $\mathcal{T}_{p}$.

While the above curvature result also shows that the fibers of $\mathcal{T}_{p}$ cannot be totally geodesic, it turns out that the images of certain sections $s: T_{p} \rightarrow \mathcal{T}_{p}$ are totally geodesic. This observation may be useful for further applications in algebraic geometry, along the line of [JY].

Let me also mention that a detailed study of $T_{p, n}$ in terms of harmonic maps has recently and independently been undertaken by my student Lohkamp in his diplom thesis and by Wolf and Wolpert.

I want to express my gratitude to Michael Wolf for explaining his results to me (some of his formulae are reproduced in Section 1, and they form the starting point of our investigation), and to Scott Wolpert for stimulating discussions, and for pointing out to me that my original statement of Theorem 2 was not entirely 
correct.

I also want to thank the referee for his excellent refereeing and his detailed suggestions.

I gratefully acknowledge the hospitality of MSRI (Berkeley) and IAS (Princeton), as well as financial support from Stiftung Volkswagenwerk and DFG (through its SFB program).

\section{Variations of conformal structures and harmonic maps}

\subsection{Variations of the image structure}

We let $\Sigma$ be a compact oriented surface of genus $p \geq 2$. We denote conformal structures on $\Sigma$ by $g, \gamma$. Each conformal structure uniquely determines a hyperbolic metric on $\Sigma$. In this paper we shall use the convention to equip each conformal structure with this hyperbolic metric.

The hyperbolic metric in $(\Sigma, g)$ will be denoted by

$$
\lambda^{2}(z) d z d \bar{z}, \quad z \text { being a conformal parameter, }
$$

and the one on $(\Sigma, \gamma)$ by

$$
\varrho^{2}(u) d u d \bar{u}, \quad u \text { again being a conformal parameter. }
$$

For a $C^{1}$-map $v=v(z):(\Sigma, g) \rightarrow(\Sigma, \gamma)$, we define the energy as

$$
E(v):=\frac{1}{2} \int_{(\Sigma, g)} \varrho^{2}(v(z))\left(v_{z} \bar{v}_{\bar{z}}+\bar{v}_{z} v_{\bar{z}}\right) d z d \bar{z}
$$

subscripts denoting partial derivatives.

Critical points of $E$ are called harmonic maps.

Harmonic maps satisfy the Euler-Lagrange equations for $E$, namely

$$
u_{z \bar{z}}+\frac{2 \varrho_{u}}{\varrho} u_{z} u_{\bar{z}}=0
$$

The following lemma summarizes results of Al'ber [Al 1,2], Eells-Sampson [ES], Hartman [H], Schoen-Yau [SY] and Sampson [Sa]:

Lemma 1. Given $g, \gamma$, there exists a unique harmonic map

$$
u(g, \gamma):(\Sigma, g) \rightarrow(\Sigma, \gamma)
$$

homotopic to the identity of $\Sigma . u(g, \gamma)$ is a diffeomorphism.

$$
\varphi d z^{2}:=\varrho^{2} u_{z} \bar{u}_{z} d z^{2}
$$


is a holomorphic quadratic differential on $(\Sigma, g)$.

$$
\varphi \equiv 0 \quad \Leftrightarrow \quad u \text { is conformal } \Leftrightarrow g=\gamma \text {. }
$$

Remark. We point out that Lemma 1 can be extended to surfaces with punctures. $\varphi d z^{2}$ then may have simple poles at the punctures. This remark justifies the claim about $T_{p, n}$ in the introduction.

In this chapter, $u=u(g, \gamma)$ will always denote a harmonic map.

We define functions

$$
\begin{aligned}
& H(u):=\left|u_{z}\right|^{2}=\frac{\varrho^{2}(u(z))}{\lambda^{2}(z)} u_{z} \bar{u}_{\bar{z}}, \\
& L(u):=\left|u_{\bar{z}}\right|^{2}=\frac{\varrho^{2}(u(z))}{\lambda^{2}(z)} u_{\bar{z}} \bar{u}_{z} .
\end{aligned}
$$

In this notation

$$
u^{*}\left(\varrho^{2} d u d \bar{u}\right)=\varphi d z^{2}+\lambda^{2}(z)(H+L) d z d \bar{z}+\bar{\varphi} d \bar{z}^{2} .
$$

To $\varphi$, we associate the harmonic Beltrami differential

$$
\mu(z):=\bar{\varphi}(z) \cdot \frac{1}{\lambda^{2}(z)} .
$$

Then

$$
H(u) L(u)=\mu \bar{\mu}
$$

One also computes, using the equation (1.1) for a harmonic $u$, that

$$
\Delta \log H(u)=-2+2(H(u)-L(u)),
$$

where $\Delta$ is the Laplace-Beltrami operator on $(\Sigma, g)$ (note that the curvatures of $\varrho^{2}(u)$ and $\lambda^{2}(z)$ are both -1$)$. References for the preceding facts are [J1] and [J2]. We observe that since $u$ is a diffeomorphism, and $H(u)-L(u)$ its Jacobian that

$$
H(u)>0
$$

so that $\log H(u)$ is well defined.

We denote the space of harmonic Beltrami differentials on $(\Sigma, g)$ by $\mathcal{H}(g)$ and identify $\mathcal{H}(g)$ with its tangent space at the origin $\mu=0$.

Following M. Wolf [Wf], we shall now study the effect of variations of $\mu$. 
We want to differentiate (1.3) and (1.4) in the variable $\mu$ at the origin $\mu=0$ in directions $\alpha, \bar{\beta}$; subscripts will denote corresponding derivatives. Then, at $\mu=0$,

$$
\begin{gathered}
H \equiv 1, \\
L \equiv 0 \\
H_{\alpha} L+H L_{\alpha}=0,
\end{gathered}
$$

and consequently

$$
\begin{gathered}
L_{\alpha} \equiv 0 \\
\Delta \frac{H_{\alpha}}{H}=2\left(H_{\alpha}-L_{\alpha}\right),
\end{gathered}
$$

and consequently

$$
\begin{aligned}
& H_{\alpha} \equiv 0, \\
& H_{\alpha \bar{\beta}} L+H_{\alpha} L_{\bar{\beta}}+H_{\bar{\beta}} L_{\alpha}+H L_{\alpha \bar{\beta}}=\alpha \bar{\beta},
\end{aligned}
$$

and consequently

$$
L_{\alpha \bar{\beta}}=\alpha \bar{\beta},
$$

$$
\Delta\left(\frac{H_{\alpha \bar{\beta}}}{H}-\frac{H_{\alpha} H_{\bar{\beta}}}{H^{2}}\right)=2\left(H_{\alpha \bar{\beta}}-L_{\alpha \bar{\beta}}\right),
$$

and consequently, defining

$$
\begin{gathered}
D:=-2(\Delta-2)^{-1}, \\
H_{\alpha \bar{\beta}}=D(\alpha \bar{\beta}) .
\end{gathered}
$$

Likewise

$$
L_{\alpha \beta}=0,
$$




$$
H_{\alpha \beta}=0 \text {. }
$$

For variations of the energy

$$
E(u)=\frac{1}{2} \int(H(u)+L(u)) \lambda^{2} d z d \bar{z},
$$

we get

$$
E_{\alpha}=0
$$

from (1.9), (1.11). Moreover, since

$$
\int D(\alpha \bar{\beta}) \lambda^{2} d z d \bar{z}=\int(\Delta-2)(\Delta-2)^{-1} \alpha \bar{\beta} \lambda^{2} d z d \bar{z}=\int \alpha \bar{\beta} \lambda^{2} d z d \bar{z}
$$

we get from (1.13), (1.16)

$$
E_{\alpha \bar{\beta}}=\int \alpha \bar{\beta} \lambda^{2} d z d \bar{z} .
$$

This means that the second variation of the energy w.r.t. the image structure yields the Weil-Petersson metric on $\mathcal{H}(g)$. Of course, we can also look dually at $Q(g)$, the space of holomorphic quadratic differentials on $(\Sigma, g)$, and obtain, putting

$$
\begin{gathered}
\psi_{1}(z)=\bar{\alpha}(z) \lambda^{-2}(z), \\
\psi_{2}(z)=\bar{\beta}(z) \lambda^{-2}(z), \\
E_{\psi_{1} \bar{\psi}_{2}}=\int \psi_{1} \bar{\psi}_{2} \frac{1}{\lambda^{2}} d z d \bar{z} .
\end{gathered}
$$

The following interpretation of the preceding discussion will be of use later on. We fix $(\Sigma, g)$. For any $(\Sigma, \gamma)$, we look at the harmonic map

$$
u=u(g, \gamma):(\Sigma, g) \rightarrow(\Sigma, \gamma)
$$

homotopic to the identity of $\Sigma$. This map yields the harmonic Beltrami differential at $(\Sigma, g)$

$$
\frac{\varrho^{2}(u)}{\lambda^{2}(z)} u_{\bar{z}} \bar{u}_{\bar{z}} d \bar{z} \otimes \frac{\partial}{\partial z} .
$$

We thus obtain a map

$$
q_{g}: T_{p} \rightarrow \mathcal{H}(g)
$$

The following result is due to Wolf ([Wf]; cf. also [J2] for a proof): 
Lemma 2. The map

$$
q_{g}: T_{p} \rightarrow \mathcal{H}(g) .
$$

from Teichmüller space to the space of harmonic Beltrami differentials at $(\Sigma, g)$ is bijective, and the transition maps

$$
q_{g_{2}} \circ q_{g_{1}}^{-1}: \mathcal{H}\left(g_{1}\right) \rightarrow \mathcal{H}\left(g_{2}\right)
$$

are smooth.

In Section 2, we shall use $\mathcal{H}(g)$ as coordinates for $T_{p}$. Actually, we shall need these coordinates only locally, and this local property can already be deduced from the injectivity of $q_{g}$ obtained in [Sa] from (1.4) as a consequence of the maximum principle.

We now represent $(\Sigma, g)$ and $(\Sigma, \gamma)$ by their fundamental regions in $H:=$ $\{z=x+i y: y>0\}$. Then $\varrho^{2}(u)=-2 /(u-\bar{u})^{2}, \lambda^{2}(z)=-2 /(z-\bar{z})^{2}$.

We can furthermore normalize the situation so that the lift of $u$ to $H, u: H \rightarrow$ $H$, fixes $0,1, \infty$.

The point of the rest of this subsection is to compare a harmonic and a quasiconformal homeomorphism between two conformal structures. We shall see that expansions in terms of $\mu \in \mathcal{H}(g)$ at $\mu=0$ agree up to second order. This will be useful in the sequel, because we need to make computations for quasiconformal maps and we can perform such computations for harmonic maps.

For $\mu \in \mathcal{H}(g)$, with $|\mu|_{L^{\infty}(H)}<1$ we let $f^{\mu}: H \rightarrow H$ be the homeomorphism solution of

$$
f_{\bar{z}}^{\mu}=\mu f_{z}^{\mu}
$$

fixing $0,1, \infty$. Proofs of the existence and uniqueness of the quasiconformal homeomorphism $f^{\mu}$ can be found in [AB], [J1], [J2].

We compare this with

$$
u_{\bar{z}}=\frac{\bar{\varphi}}{\lambda^{2} H} u_{z}=\frac{\bar{\varphi} \cdot y^{2}}{H} u_{z} .
$$

With $\mu:=\bar{\varphi} y^{2} \in \mathcal{H}(g)$, we thus have

$$
u_{\bar{z}}=\frac{\mu}{H} u_{z} .
$$

Again, we take derivatives at $\mu=0$, and denote derivatives in the direction $\alpha$ by $\dot{u}[\alpha], \dot{f}[\alpha]$, etc.

Then, at $\mu=0$,

$$
\dot{f}[\alpha]_{\bar{z}}=\alpha f_{z},
$$




$$
\ddot{f}[\alpha, \beta]_{\bar{z}}=\alpha \dot{f}[\beta]_{z}+\beta \dot{f}[\alpha]_{z} .
$$

Likewise, at $\mu=0$,

$$
\begin{gathered}
\dot{u}[\alpha]_{\bar{z}}=\frac{\alpha}{H} u_{z}=\alpha u_{z}, \\
\ddot{u}[\alpha, \beta]_{\bar{z}}=\frac{\alpha}{H} \dot{u}[\beta]_{z}+\frac{\beta}{H} \dot{u}[\alpha]_{z}=\alpha \dot{u}[\beta]_{z}+\beta \dot{u}[\alpha]_{z},
\end{gathered}
$$

using (1.6) and (1.11).

Therefore, the differential equations for $u$ and $f$ agree to second order, and hence, because both $f^{\mu}$ and $u$ are unique under the present normalizations, near $\mu=0, f^{\mu}$ and $u$ agree as well up to second order.

In particular, with

$$
\begin{aligned}
H^{f} & :=\frac{(z-\bar{z})^{2}}{(f-\bar{f})^{2}} f_{z} \bar{f}_{\bar{z}} \\
L^{f} & :=\frac{(z-\bar{z})^{2}}{(f-\bar{f})^{2}} \bar{f}_{z} f_{\bar{z}}
\end{aligned}
$$

we have at $\mu=0$

$$
L_{\alpha}^{f}=0=H_{\alpha}^{f},
$$

$$
L_{\alpha \bar{\beta}}^{f}=\alpha \bar{\beta}
$$

$$
H_{\alpha \bar{\beta}}^{f}=H_{\alpha \bar{\beta}}=D(\alpha \bar{\beta})
$$

by (1.16), and likewise, using (1.18)

$$
L_{\alpha \beta}^{f}=0=H_{\alpha \beta}^{f} .
$$

For later applications, we point out that $H^{f}-L^{f}$ represents the Jacobian of $f$, i.e. the ratio of the $f$-pullback of the hyperbolic area element and the hyperbolic area element of the domain. 


\subsection{Variations of the domain structure}

The groups $\Gamma, \Gamma^{t}$ occurring in the sequel will be discrete subgroups of $\mathrm{PL}(2 \mathrm{R})$ (the automorphism group of the upper halfplane), homomorphic to $\pi_{1}(\Sigma)$.

We continue to use the notations of 1.1.

We consider the energy $E$ as a function of the conformal structure $g$ by always computing the energy of the harmonic map $u=u(g, \gamma):(\Sigma, g) \rightarrow(\Sigma, \gamma)$ ( $\gamma$ is fixed in this subsection). Thus $E=E(u(g, \gamma), g \gamma)$.

We represent $(\Sigma, g)$ by a quotient $H / \Gamma$ of the upper half plane. The plan is to represent variations of the conformal (= hyperbolic, by our convention) structure by variations of $\Gamma=\Gamma^{0}$. The elements of the new group $\Gamma^{t}(t \neq 0)$ differ from those of $\Gamma$ by an equivariant (quasiconformal) diffeomorphism $\zeta_{t}((1.33)$ below). If $u$ is a map with domain $H / \Gamma, u \circ \zeta_{t}^{-1}$ is a map with domain $H / \Gamma^{t}$. We compute the energy of $u \circ \zeta_{t}^{-1}$. If $u$ is harmonic, $u \circ f_{t}^{-1}$ need no longer be harmonic. This will not matter for first order computations at $t=0$, since the first derivative of $E$ w.r.t. $u$ vanishes because $u$ as a harmonic map is a critical point of $E$.

We now start by deriving the formula for the energy of $u \circ \zeta_{t}^{-1}$.

We thus let $\zeta_{t}: H \rightarrow H$ be a family of diffeomorphisms, depending smoothly on $t$, with $\zeta_{0}=\mathrm{id}$. We require for every $t$ that $\zeta_{t}$ is equivariant in the following sense: There exists a group $\Gamma^{t}$, and for every $\sigma \in \Gamma$ for some $\sigma^{t} \in \Gamma^{t}$, with

$$
\zeta_{t} \circ \sigma=\sigma^{t} \circ \zeta_{t}
$$

We let

$$
\omega:={\frac{\partial \zeta_{t}}{\partial t}}_{\mid t=0}, \quad\left(\Sigma, g_{t}\right):=H / \Gamma^{t},
$$

and as before, for a harmonic map $u:(\Sigma, g) \rightarrow(\Sigma, \gamma), \varphi=\varrho^{2}(u) u_{z} \bar{u}_{z}$. We compute from the chain rule, with $\zeta=\zeta_{t}$,

$$
\begin{aligned}
E\left(u \circ \zeta_{t}^{-1}, g_{t}, \gamma\right)= & \frac{1}{2} \int_{H / \Gamma} \varrho^{2}\left\{\left(u_{z} \bar{u}_{\bar{z}}+\bar{u}_{z} u_{\bar{z}}\right)\left(\zeta_{z} \bar{\zeta}_{\bar{z}}+\bar{\zeta}_{z} \zeta_{\bar{z}}\right)\right. \\
& \left.-2 u_{z} \bar{u}_{z} \zeta_{\bar{z}} \bar{\zeta}_{\bar{z}}-2 \bar{u}_{\bar{z}} u_{\bar{z}} \zeta_{z} \bar{\zeta}_{z}\right\} \frac{d z d \bar{z}}{\zeta_{z} \bar{\zeta}_{\bar{z}}-\bar{\zeta}_{z} \zeta_{\bar{z}}}
\end{aligned}
$$

and

$$
\begin{aligned}
\frac{d}{d t} E & \left(u \circ \zeta_{t}^{-1}, g_{t}, \gamma\right)_{\mid t=0} \\
= & \frac{1}{2} \int_{H / \Gamma} \varrho^{2}\left\{\left(u_{z} \bar{u}_{\bar{z}}+\bar{u}_{z} u_{\bar{z}}\right)\left(\omega_{z} \bar{\zeta}_{\bar{z}}+\zeta_{z} \bar{\omega}_{\bar{z}}+\bar{\omega}_{z} \zeta_{\bar{z}}+\omega_{\bar{z}} \bar{\zeta}_{z}\right)\right. \\
& -2 u_{z} \bar{u}_{z}\left(\omega_{\bar{z}} \bar{\zeta}_{\bar{z}}+\zeta_{\bar{z}} \bar{\omega}_{\bar{z}}\right)-2 u_{\bar{z}} \bar{u}_{\bar{z}}\left(\omega_{z} \bar{\zeta}_{z}+\zeta_{z} \bar{\omega}_{z}\right) \frac{1}{\zeta_{z} \bar{\zeta}_{\bar{z}}-\bar{\zeta}_{z} \zeta_{\bar{z}}}
\end{aligned}
$$




$$
\begin{aligned}
& -\left(\omega_{z} \bar{\zeta}_{\bar{z}}+\zeta_{z} \bar{\omega}_{\bar{z}}-\omega_{\bar{z}} \bar{\zeta}_{z}-\zeta_{\bar{z}} \bar{\omega}_{z}\right) \cdot \\
& \cdot\left(\left(u_{z} \bar{u}_{\bar{z}}+\bar{u}_{z} u_{\bar{z}}\right)\left(\zeta_{z} \bar{\zeta}_{\bar{z}}+\zeta_{\bar{z}} \bar{\zeta}_{z}\right)-2 u_{z} \bar{u}_{z} \zeta_{\bar{z}} \bar{\zeta}_{\bar{z}}-2 u_{\bar{z}} \bar{u}_{\bar{z}} \zeta_{z} \bar{\zeta}_{z}\right) \\
& \left.\cdot \frac{1}{\left(\zeta_{z} \bar{\zeta}_{\bar{z}}-\bar{\zeta}_{z} \zeta_{\bar{z}}\right)^{2}}\right\} d z d \bar{z} \\
= & \left.-\int\left(\varrho^{2} u_{z} \bar{u}_{z} \omega_{\bar{z}}+\varrho^{2} u_{\bar{z}} \bar{u}_{\bar{z}} \bar{\omega}_{z}\right) d z d \bar{z}, \quad \text { (since at } t=0, \zeta=z\right) \\
= & -2 \operatorname{Re} \int \varphi \omega_{\bar{z}} d z d \bar{z} .
\end{aligned}
$$

In order to apply (1.35), we have to relate our two ways of varying $(\Sigma, g)$, one by varying the group $\Gamma$, the other by expressing variations through harmonic Beltrami differentials. We now do this.

An element $\alpha \in \mathcal{H}(g)$ transforms via

$$
(\alpha \circ \sigma) \bar{\sigma}_{\bar{z}}=\alpha \sigma_{z} \quad \text { for } \quad \sigma \in \Gamma
$$

We now equip $H$ with two hyperbolic metrics, $y^{-2} d z d \bar{z}$ and $y^{-2}|d z+t \alpha d \bar{z}|^{2}$, and we let

$$
\zeta_{t}:\left(H, \frac{1}{y^{2}} d z d \bar{z}\right) \rightarrow\left(H, \frac{1}{y^{2}}|d z+t \alpha d \bar{z}|^{2}\right)
$$

be conformal, normalized with

$$
\zeta_{0}=z
$$

Then,

$$
\zeta_{t, \bar{z}}=t \alpha \zeta_{t, z}
$$

hence

$$
\omega_{\bar{z}}=\frac{\partial}{\partial t} \zeta_{t, \bar{z} \mid t=0}=\alpha
$$

(1.36) implies that with $\zeta_{t}$, also $\zeta_{t} \circ \sigma$ solves (1.38) for $\sigma \in \Gamma$. Hence there exists a conformal $\sigma^{t}$ with

$$
\zeta_{t} \circ \sigma=\sigma^{t} \circ \zeta_{t}, \quad \sigma^{t} \in \Gamma^{t} \text {. }
$$

We have thus related the two ways of varying $(\Sigma, g) . \alpha \in \mathcal{H}(g)$ defines the family of diffeomorphisms $\zeta_{t}$, and then a family of groups $\Gamma^{t}$, and vice versa. We are now ready to study variations of $E$ as a function of the domain structure $g$ in the direction $\alpha \in \mathcal{H}(g)$. We use the above correspondence between $\alpha$ and the families $\zeta_{t}, \Gamma^{t}$, and we denote the conformal structure of $H / \Gamma^{t}$ by $g_{t}$. The 
dependence of $E$ on $g$ is twofold, because the harmonic map $u=u(g, \gamma):(\Sigma, g) \rightarrow$ $(\Sigma, \gamma)$ also depends on $g$.

Since $u$ is harmonic, however,

$$
\frac{\partial}{\partial u} E(u, g, \gamma)=0
$$

hence

$$
\begin{aligned}
& \left\langle\frac{\partial}{\partial g} E((u(g, \gamma), g, \gamma), \alpha\rangle\right. \\
& \quad=\frac{\partial}{\partial t} E\left(\left(u(g, \gamma) \circ \zeta_{t}^{-1}, g_{t}, \gamma\right)_{\mid t=0}=-2 \operatorname{Re} \int \varphi \alpha d z d \bar{z}\right.
\end{aligned}
$$

from (1.35). Since $\operatorname{Re} \int \varphi \alpha d z d \bar{z}$ is a nondegenerate pairing between $Q(g)$ and $\mathcal{H}(g)$, we see that $g$ is a critical point of $E$

$$
\Longleftrightarrow \varphi \equiv 0 \Longleftrightarrow u \text { is conformal } \Longleftrightarrow g=\gamma .
$$

We now want to study second variations of $E$ at $g=\gamma$. We continue to identify $(\Sigma, g)$ with $H / \Gamma$, and the map $u:(\Sigma, g) \rightarrow(\Sigma, \gamma)$ is considered as a normalized equivariant map $u: H \rightarrow H$, and the energy then has to be evaluated on a fundamental region. If $g=\gamma$, we have $u=\mathrm{id}$. Since $H$ is a subset of the linear space $\mathbf{C}$, we can write variations of $u$ as $u+t h$. As always, $t$ varies in a small neighborhood of 0 .

As $u$ is a diffeomorphism, a variation $u+t h$ amounts to the same as studying variations $u \circ \zeta_{t}$ (if $|t|$ is sufficiently small).

We put

$$
\varphi_{t}:=\varrho^{2}(u+t h)(u+t h)_{z}(\bar{u}+t \bar{h})_{\bar{z}}
$$

and since $u$ is conformal

$$
\frac{\partial}{\partial t} \varphi_{t_{\mid t=0}}=\varrho^{2}(u) u_{z} \bar{h}_{z}
$$

Thus

$$
\frac{d}{d t}\left\langle\frac{\partial}{\partial g} E(u+t h, g, \gamma), \alpha\right\rangle_{\mid t=0}=-2 \int \operatorname{Re} \varrho^{2} u_{z} \bar{h}_{z} \alpha d z d \bar{z}=2 \int \operatorname{Re} \bar{h}_{z} \alpha \frac{1}{y^{2}} d z d \bar{z}
$$

as in our setting, $u$, since conformal, is the identity

$$
=0, \text { since } \alpha \in \mathcal{H}(g)
$$

(see (2.7) for this point). 
This means that, at $g=\gamma$,

$$
\frac{\partial}{\partial u} \frac{\partial}{\partial g} E(u(g, \gamma), g, \gamma)=0 .
$$

A variation $\alpha$ of $g$ induces a variation $u_{\alpha}$ of $u$. As $u(g, \gamma)$ is harmonic

$$
\frac{\partial}{\partial u} E(u(g, \gamma), g, \gamma)=0
$$

for all $g$. Hence

$$
\begin{aligned}
0 & =\left\langle\frac{\partial}{\partial g} \frac{\partial}{\partial u} E(u(g, \gamma), g, \gamma), \alpha\right\rangle \\
& =\left\langle\frac{\partial^{2}}{\partial u^{2}} E(u(g, \gamma), g, \gamma) u_{\alpha}, \alpha\right\rangle+\left\langle\frac{\partial}{\partial u} \frac{\partial}{\partial g} E(u(g, \gamma), g, \gamma), \alpha\right\rangle \\
& =\left\langle\frac{\partial^{2}}{\partial u^{2}} E(u(g, \gamma), g, \gamma) u_{\alpha}, \alpha\right\rangle
\end{aligned}
$$

by (1.41). Since $\left(\partial^{2} / \partial u^{2}\right) E$ is strictly positive definite, because $\gamma$ has negative curvature, we conclude, again at $g=\gamma$,

$$
u_{\alpha}=0 \text {. }
$$

The preceding result was obtained by Tromba ([2]) by a different method.

One can also use the present method to compute second variations of $E$ w.r.t. the domain structure. Since we do not need the corresponding formula in the present paper, we refer to [J2].

\section{The Weil-Petersson metric}

\subsection{Preliminaries}

From Section 1, (1.22), we recall that the Weil-Petersson metric can be computed from the formula for the second variation of the energy of a harmonic map w.r.t. the image metric, namely

$$
h_{\alpha \bar{\beta}}:=\int_{(\Sigma, g)} \alpha \bar{\beta} \lambda^{2} d z d \bar{z}=E_{\alpha \bar{\beta}}
$$

for $\alpha, \beta \in \mathcal{H}(g)$, considered as a complex vector space.

Likewise, we get an induced metric on $Q(g)$, e.g. for $\varphi, \psi \in Q(g)$

$$
\langle\varphi, \psi\rangle_{\mathbf{C}}=\int_{(\Sigma, g)} \varphi \bar{\psi} \frac{1}{\lambda^{2}} d z d \bar{z}
$$


We now want to study derivatives of the Weil-Petersson metric at the origin of our coordinate charts.

In the sequel, we shall use the fact that infinitesimal diffeomorphisms are $L^{2}$-orthogonal to the harmonic Beltrami differentials in a similar way as Ahlfors [A1], [A2] and Wolpert [Wp], but otherwise our considerations will be conceptually different from theirs.

Studying derivatives of $h_{\alpha \bar{\beta}}$ involves changing the base surface $(\Sigma, g)$. We recall that Lemma 2 allows us to use $\mathcal{H}(g)$ as global coordinates for Teichmüller space $T_{p}$. In these coordinates, $(\Sigma, g)$ corresponds to the origin. We shall now employ these coordinates. Since $\mathcal{H}(g)$ is a linear space, we can identify it with its tangent space at 0 . Coordinate representations of points in $T_{p}$ shall be denoted by $\mu, \nu, \varrho, \sigma, \ldots$, where as tangent vectors at the origin will be denoted by $\alpha$, $\beta, \gamma, \ldots$

For $\mu, \nu \in \mathcal{H}(g)$, we let

$$
\begin{aligned}
& w: \mu \rightarrow \mu+\nu, \\
& \zeta: 0 \rightarrow \mu, \\
& u: 0 \rightarrow \mu+\nu
\end{aligned}
$$

be harmonic maps.

We also put

$$
v:=u \circ \zeta^{-1}
$$

and $z$ will denote a complex parameter on $(\Sigma, g)$, the conformal structure represented by $0 \in \mathcal{H}(g)$.

We recall that in order to obtain $h_{\alpha \bar{\beta}}(\mu)$, we have to look at the harmonic map $w: \mu \rightarrow \mu+\nu$ and differentiate the $\nu$-dependence of its energy at $\nu=0$ in the directions $\alpha$ and $\bar{\beta}$. In order to obtain derivatives $h_{\alpha \bar{\beta}, \gamma}(0)$ at the origin, we then have to differentiate the $\mu$-dependence at $\mu=0$ in the direction $\gamma$, and likewise for second derivatives. It is the main purpose of this section to compute first and second derivatives of $h_{\alpha \bar{\beta}}$ at the origin.

If $h(\varrho, \sigma): \varrho \rightarrow \sigma$ is a map, we shall denote differentiation w.r.t. the image variable $\sigma$ by ', and w.r.t. the domain variable by $\cdot$. The corresponding tangent vectors will be given in brackets; the image direction always comes first.

For example, $\dot{h}^{\prime}[\bar{\beta}]\langle\gamma\rangle$ denotes differentiation of the $\sigma$-dependence in the direction $\bar{\beta}$ and of the $\varrho$ dependence in the direction $\gamma$.

We denote the hyperbolic metric on $\mu$ by $\ell^{2}(\zeta) d \zeta d \bar{\zeta}$. In this notation

$$
h_{\alpha \bar{\beta}}(\mu)=\int\left(\frac{\varrho^{2}(w)}{\ell^{2}(\zeta)} w_{\bar{\zeta}} \bar{w}_{\zeta}\right)^{\prime \prime}[\alpha, \bar{\beta}] \ell^{2}(\zeta) d \zeta d \bar{\zeta}=\int w_{\bar{\zeta}}^{\prime}[\alpha] \bar{w}_{\zeta}^{\prime}[\bar{\beta}] \ell^{2}(\zeta) d \zeta d \bar{\zeta}
$$

where all quantities are evaluated on the surface corresponding to $\mu$. 
In the rest of this subsection, we derive the well-known fact that the harmonic Beltrami differentials are $L^{2}$-orthogonal to the infinitesimal diffeomorphisms of the base surface as well as some consequences.

We let $d_{t}: \mu \rightarrow \mu$ be a family of diffeomorphisms, depending smoothly on $t$, with $d_{0}=$ id. As before, we look at the lifts $d_{t}: H \rightarrow H$ which then are $\Gamma$-equivariant, where $\mu=H / \Gamma$ :

$$
d(\chi \zeta)=\chi d(\zeta)
$$

for $\chi \in \Gamma, \zeta \in H$.

The equation (2.5) implies

$$
\frac{\partial}{\partial t} d_{t}(\chi \zeta)=\chi \frac{\partial}{\partial t} d_{t}(\zeta)
$$

(Of course, $\chi$ here acts on a vector field on $H$, i.e. by pullback.)

The claim that the Beltrami differentials are $L^{2}$-orthogonal to the infinitesimal diffeomorphisms, i.e.

$$
\int \alpha \frac{\partial}{\partial t} d_{t, \zeta} \ell^{2}(\zeta) d \zeta d \bar{\zeta}=0 \quad \text { for } \quad \alpha \in \mathcal{H}(\mu)
$$

now follows by integrating (2.7) by parts, namely

$$
\int \frac{\partial}{\partial \zeta}\left(\ell^{2}(\zeta) \alpha\right) \frac{\partial}{\partial t} d_{t} d \zeta d \bar{\zeta}
$$

which is valid because of (2.6), and this vanishes since $\alpha \in \mathcal{H}(\mu)$.

We shall put this to use as follows:

We recall that

$$
w_{\bar{\zeta}}^{\prime}[\alpha]=\alpha \in \mathcal{H}(\mu)
$$

cf. (1.27), and we let now $d_{\nu}: \mu \rightarrow \mu$ be a family of diffeomorphisms depending on $\nu \in \mathcal{H}(\mu)$, again with $d_{0}=$ id. We let

$$
\frac{\partial}{\partial \nu} d[\bar{\beta}]
$$

be the $\nu$-derivative of $d$ in the direction $\bar{\beta}$ at $\nu=0$. Then (2.7) yields

$$
\int w_{\bar{\zeta}}^{\prime}[\alpha] \frac{\partial}{\partial \nu} d_{\zeta}[\bar{\beta}] \ell^{2}(\zeta) d \zeta d \bar{\zeta}=0
$$

This implies

$$
\int w_{\bar{\zeta}}^{\prime}[\alpha] \overline{w \circ d_{\zeta}^{\prime}}[\bar{\beta}] \ell^{2}(\zeta) d \zeta d \bar{\zeta}=\int w_{\bar{\zeta}}^{\prime}[\alpha] \bar{w}_{\zeta}^{\prime}[\bar{\beta}] \ell^{2}(\zeta) d \zeta d \bar{\zeta}
$$

and in particular

$$
\int w_{\bar{\zeta}}^{\prime}[\alpha] \bar{w}_{\zeta}^{\prime}[\bar{\beta}] \ell^{2}(\zeta) d \zeta d \bar{\zeta}=\int w_{\bar{\zeta}}^{\prime}[\alpha] \bar{v}_{\zeta}^{\prime}[\bar{\beta}] \ell^{2}(\zeta) d \zeta d \bar{\zeta},
$$

with $v=u \circ \zeta^{-1}$ as above. 


\subsection{Differentiation of the harmonic map equation}

In order to study derivatives of the energy, we have to study the variation of a harmonic map in its dependence on the conformal structure of domain and image. For this purpose, we have to differentiate the differential equation that $w: \mu \rightarrow \mu+\nu$ as a harmonic map has to satisfy

$$
w_{\zeta \bar{\zeta}}-\frac{2}{(w-\bar{w})} w_{\zeta} w_{\bar{\zeta}}=0
$$

This is equivalent to, writing now $w(\zeta)=\omega(z(\zeta))$

$$
\begin{aligned}
& \omega_{z} z_{\zeta \bar{\zeta}}+\omega_{\bar{\zeta}} \bar{z}_{\zeta \bar{\zeta}}+\left(\omega_{z z}-\frac{2}{\omega-\bar{\omega}} \omega_{z} \omega_{z}\right) z_{\zeta} z_{\bar{\zeta}} \\
& \quad+\left(\omega_{\bar{z} \bar{z}}-\frac{2}{\omega-\bar{\omega}} \omega_{\bar{z}} \omega_{\bar{z}}\right) \bar{z}_{\zeta} \bar{z}_{\bar{\zeta}}+\left(\omega_{z \bar{z}}-\frac{2}{\omega-\bar{\omega}} \omega_{z} \omega_{\bar{z}}\right)\left(z_{\zeta} \bar{z}_{\bar{\zeta}}+\bar{z}_{\zeta} z_{\bar{\zeta}}\right)=0
\end{aligned}
$$

From the chain rule, one computes

$$
\begin{aligned}
z_{\zeta \bar{\zeta}}= & \frac{1}{\left(\zeta_{z} \bar{\zeta}_{\bar{z}}-\zeta_{\bar{z}} \bar{\zeta}_{z}\right)^{3}}\left(\zeta_{z z} \bar{\zeta}_{\bar{z}} \bar{\zeta}_{\bar{z}} \zeta_{\bar{z}}+\zeta_{\bar{z} \bar{z}} \bar{\zeta}_{\bar{z}} \bar{\zeta}_{z} \zeta_{z}\right. \\
& \left.-\bar{\zeta}_{z z} \zeta_{\bar{z}} \zeta_{\bar{z}} \bar{\zeta}_{\bar{z}}-\bar{\zeta}_{\bar{z} \bar{z}} \zeta_{\bar{z}} \bar{\zeta}_{z} \zeta_{z}+\left(\bar{\zeta}_{z \bar{z}} \zeta_{\bar{z}}-\zeta_{\bar{z} z} \bar{\zeta}_{\bar{z}}\right)\left(\zeta_{z} \bar{\zeta}_{\bar{z}}+\zeta_{\bar{z}} \bar{\zeta}_{z}\right)\right)
\end{aligned}
$$

and (2.11) becomes

$$
\begin{aligned}
0= & \omega_{z} \frac{1}{\zeta_{z} \bar{\zeta}_{\bar{z}}-\zeta_{\bar{z}} \bar{\zeta}_{z}}\left(\zeta_{z z} \bar{\zeta}_{\bar{z}} \bar{\zeta}_{\bar{z}} \zeta_{\bar{z}}+\zeta_{\bar{z} \bar{z}} \bar{\zeta}_{\bar{z}} \bar{\zeta}_{z} \zeta_{z}\right. \\
& -\bar{\zeta}_{z z} \zeta_{\bar{z}} \zeta_{\bar{z}} \bar{\zeta}_{\bar{z}}-\bar{\zeta}_{\bar{z} \bar{z}} \zeta_{\bar{z}} \bar{\zeta}_{z} \zeta_{z} \\
& +\left(\left(\bar{\zeta}_{z \bar{z}} \zeta_{\bar{z}}-\zeta_{\bar{z} z} \bar{\zeta}_{\bar{z}}\right)\left(\zeta_{z} \bar{\zeta}_{\bar{z}}+\zeta_{\bar{z}} \bar{\zeta}_{z}\right)\right) \\
& +\omega_{\bar{z}} \frac{1}{\zeta_{z} \bar{\zeta}_{\bar{z}}-\zeta_{\bar{z}} \bar{\zeta}_{z}}\left(\bar{\zeta}_{\bar{z} \bar{z}} \zeta_{z} \zeta_{z} \bar{\zeta}_{z}+\bar{\zeta}_{z z} \zeta_{z} \zeta_{\bar{z}} \bar{\zeta}_{\bar{z}}\right. \\
& -\zeta_{\bar{z} \bar{z} \bar{\zeta}_{z}} \bar{\zeta}_{z} \zeta_{z}-\zeta_{z z} \bar{\zeta}_{z} \zeta_{\bar{z}} \bar{\zeta}_{\bar{z}} \\
& \left.+\left(\zeta_{\bar{z} z} \bar{\zeta}_{z}-\bar{\zeta}_{z \bar{z}} \zeta_{z}\right)\left(\zeta_{z} \bar{\zeta}_{\bar{z}}+\zeta_{\bar{z}} \bar{\zeta}_{z}\right)\right) \\
- & \left(\omega_{z z}-\frac{2}{\omega-\bar{\omega}} \omega_{z} \omega_{z}\right) \zeta_{\bar{z}} \bar{\zeta}_{\bar{z}}-\left(\omega_{\bar{z} \bar{z}}-\frac{2}{\omega-\bar{\omega}} \omega_{\bar{z}} \omega_{\bar{z}}\right) \zeta_{z} \bar{\zeta}_{z} \\
& +\left(\omega_{z \bar{z}}-\frac{2}{\omega-\bar{\omega}} \omega_{z} \omega_{\bar{z}}\right)\left(\zeta_{z} \bar{\zeta}_{\bar{z}}+\zeta_{\bar{z}} \bar{\zeta}_{z}\right) .
\end{aligned}
$$

We recall that $w$ goes from $\mu$ to $\mu+\nu$; therefore the $\mu$-dependence of $w$ and hence also of $\omega$ is two-fold. 
We now take the total $\mu$-derivative of (2.12) and evaluate at $\mu=\nu=0$. We recall $\zeta_{\bar{z}}=\mu \zeta_{z}$, and hence, at $\mu=0, \zeta_{\bar{z}}^{\prime}[\gamma]=\gamma \zeta_{z}=\gamma$ and $\bar{\zeta}_{z}^{\prime}[\gamma]=0$. We then obtain, using that at $\mu=\nu=0, \omega(z)=z$

$$
0=-\omega_{z} \gamma_{z}+\frac{2}{\omega-\bar{\omega}} \omega_{z} \omega_{z} \gamma+\dot{\omega}_{\bar{z} z}\langle\gamma\rangle-\frac{2}{\omega-\bar{\omega}} \omega_{z} \dot{\omega}_{\bar{z}}\langle\gamma\rangle+\omega_{\bar{z} z}^{\prime}[\gamma]-\frac{2}{\omega-\bar{\omega}} \omega_{z} \omega_{\bar{z}}^{\prime}[\gamma] .
$$

We shall now use this equation to conclude the vanishing of $\dot{\omega}\langle\gamma\rangle$ and likewise $\dot{\omega}\langle\bar{\delta}\rangle$. First $\omega_{\bar{z}}^{\prime}[\gamma]=\gamma$, and since $\gamma$ is a harmonic Beltrami differential,

$$
\gamma_{z}-\frac{2}{z-\bar{z}} \gamma=0
$$

Hence

$$
0=\dot{\omega}_{\bar{z} z}\langle\gamma\rangle-\frac{2}{z-\bar{z}} \dot{\omega}_{\bar{z}}\langle\gamma\rangle
$$

i.e. $\dot{\omega}_{\bar{z}}\langle\gamma\rangle$ is a harmonic Beltrami differential.

On the other hand, $\dot{\omega}_{\bar{z}}\langle\gamma\rangle$ represents an infinitesimal diffeomorphism of our base surface 0 , and is hence orthogonal to all harmonic Beltrami differentials, cf. (2.7).

Consequently,

$$
\dot{\omega}_{\bar{z}}\langle\gamma\rangle=0 .
$$

Likewise, we obtain at $\mu=\nu=0$, by differentiating (2.12) w.r.t. $\bar{\delta}$

$$
0=\dot{\omega}_{\bar{z} z}\langle\bar{\delta}\rangle-\frac{2}{z-\bar{z}} \dot{\omega}_{\bar{z}}\langle\delta\rangle+\omega_{\bar{z} z}^{\prime}[\bar{\delta}]-\frac{2}{z-\bar{z}} \omega_{\bar{z}}^{\prime}[\bar{\delta}] .
$$

Actually, $\omega_{\bar{z}}^{\prime}[\delta]=0$ as $\omega_{\bar{z}}^{\prime}$ is holomorphic in $\delta$; hence $\dot{\omega}_{\bar{z}}\langle\bar{\delta}\rangle$ is harmonic, and thus again

$$
\dot{\omega}_{\bar{z}}\langle\bar{\delta}\rangle=0
$$

Of course, (2.14) and (2.15) are equivalent to (1.44), as a consequence of the Riemann-Roch theorem. Namely, (2.14) and (2.15) imply that $\dot{\omega}\langle\delta\rangle$ and $\dot{\omega}\langle\bar{\delta}\rangle$ are holomorphic vector fields and thus have to vanish since $p>1$.

We want to compute $\dot{\omega}_{\bar{z}}^{\prime}[\alpha]\langle\bar{\delta}\rangle$ and $\dot{\omega}_{\bar{z}}^{\prime}[\bar{\beta}]\langle\bar{\delta}\rangle$ at $\mu=\nu=0$.

Corresponding differentiation of (2.12) yields, using $\dot{\omega}\langle\bar{\delta}\rangle=0$,

$$
\begin{aligned}
0 & =-\omega_{\bar{z}}^{\prime}[\alpha] \bar{\delta}_{\bar{z}}-\omega_{\bar{z} z}^{\prime}[\alpha] \delta+\dot{\omega}_{\bar{z} z}^{\prime}[\alpha]\langle\bar{\delta}\rangle-\frac{2}{z-\bar{z}} \dot{\omega}_{\bar{z}}^{\prime}[\alpha]\langle\bar{\delta}\rangle \\
& =-(\alpha \bar{\delta})_{\bar{z}}+\dot{\omega}_{\bar{z} z}^{\prime}[\alpha]\langle\bar{\delta}\rangle-\frac{2}{z-\bar{z}} \dot{\omega}_{\bar{z}}^{\prime}[\alpha]\langle\bar{\delta}\rangle
\end{aligned}
$$


Harmonic maps and curvature computations in Teichmüller theory

since $\omega_{\bar{z}}^{\prime}[\alpha]=\alpha$, and, using $\omega_{\bar{z}}^{\prime}[\bar{\beta}]=0$

$$
0=\dot{\omega}_{\bar{z} z}^{\prime}[\bar{\beta}]\langle\bar{\delta}\rangle-\frac{2}{z-\bar{z}} \dot{\omega}_{\bar{z}}^{\prime}[\bar{\beta}]\langle\bar{\delta}\rangle .
$$

We now investigate the transformation behaviour of $\dot{\omega}^{\prime}$; we have, for arbitrary $\mu, \nu$

$$
\omega(\chi z)=\chi^{\nu} \omega(z)
$$

where $\chi^{\nu}$ is an element of the group $\Gamma^{\nu}$, the group of automorphisms of $H$ corresponding to $\nu$, as in 1.2 .

Hence, at $\mu=\nu=0$

$$
\dot{\omega}^{\prime}(\chi z)=\chi^{\prime} \dot{\omega}(z)+\chi \dot{\omega}^{\prime}(z)=\chi \dot{\omega}^{\prime}(z),
$$

since $\dot{\omega}=0$. Therefore, $\dot{\omega}^{\prime}$ transforms as an infinitesimal diffeomorphism (as in (2.6)), and therefore, by (2.7), $\dot{\omega}_{\bar{z}}^{\prime}$ is $L^{2}$-orthogonal to the harmonic Beltrami differentials. (2.17) therefore implies

$$
\dot{\omega}_{\bar{z}}[\bar{\beta}]\langle\bar{\delta}\rangle=0 .
$$

Similarly, we compute

$$
\begin{aligned}
0= & -\omega_{z}^{\prime}[\alpha] \gamma_{z}-\omega_{z z}^{\prime}[\alpha] \gamma+\frac{4}{z-\bar{z}} \omega_{z}^{\prime}[\alpha] \gamma-\frac{2}{(z-\bar{z})^{2}}\left(\omega^{\prime}[\alpha]-\bar{\omega}^{\prime}[\alpha]\right) \gamma \\
& +\dot{\omega}_{\bar{z} z}^{\prime}[\alpha]\langle\gamma\rangle-\frac{2}{z-\bar{z}} \dot{\omega}_{\bar{z}}^{\prime}[\alpha]\langle\gamma\rangle \\
= & -\omega_{z z}^{\prime}[\alpha] \gamma+\frac{2}{z-\bar{z}} \omega_{z}^{\prime}[\alpha] \gamma-\frac{2}{(z-\bar{z})^{2}}\left(\omega^{\prime}[\alpha]-\bar{\omega}^{\prime}[\alpha]\right) \gamma \\
& +\dot{\omega}_{\bar{z} z}^{\prime}[\alpha]\langle\gamma\rangle-\frac{2}{z-\bar{z}} \dot{\omega}_{\bar{z}}^{\prime}[\alpha]\langle\gamma\rangle
\end{aligned}
$$

since $\gamma$ is harmonic, i.e. $\gamma_{z}=2 \gamma /(z-\bar{z})$.

Moreover, at $\mu=\nu=0$, from (1.11)

$$
0=H_{\alpha}=\left(\frac{(z-\bar{z})^{2}}{(\omega-\bar{\omega})^{2}} \omega_{z} \bar{\omega}_{\bar{z}}\right)^{\prime}[\alpha]
$$

yielding

$$
0=\omega_{z}^{\prime}[\alpha]+\bar{\omega}_{\bar{z}}^{\prime}[\alpha]-\frac{2}{(z-\bar{z})}\left(\omega^{\prime}[\alpha]-\bar{\omega}^{\prime}[\alpha]\right)
$$


and upon differentation, since $\bar{\omega}_{z}^{\prime}[\alpha]=0$,

$$
0=\omega_{z z}^{\prime}[\alpha]-\frac{2}{(z-\bar{z})} \omega_{z}^{\prime}[\alpha]+\frac{2}{(z-\bar{z})^{2}}\left(\omega^{\prime}[\alpha]-\bar{\omega}^{\prime}[\alpha]\right),
$$

so that $(2.21)$ gives

and then as before

$$
\dot{\omega}_{\bar{z} z}^{\prime}[\alpha]\langle\gamma\rangle-\frac{2}{z-\bar{z}} \dot{\omega}_{\bar{z}}^{\prime}[\alpha]\langle\gamma\rangle=0
$$

$$
\dot{\omega}_{\bar{z}}^{\prime}[\alpha]\langle\gamma\rangle=0,
$$

and with a similar derivation, using in addition $\bar{\omega}_{z}^{\prime}[\bar{\beta}]=\bar{\beta}$ and

$$
\bar{\beta}_{\bar{z}}+\frac{2}{z-\bar{z}} \bar{\beta}=0
$$

since $\beta$ is harmonic, we also obtain

$$
\dot{\omega}_{\bar{z}}^{\prime}[\bar{\beta}]\langle\gamma\rangle=0 .
$$

We now want to compute $\dot{\omega}_{\bar{z}}^{\prime}[\alpha]\langle\bar{\delta}\rangle$ from (2.16) and the fact that $\dot{\omega}_{\bar{z}}^{\prime}[\alpha]\langle\bar{\delta}\rangle$ is orthogonal to the harmonic Beltrami differentials.

We write $(2.16)$ as

$$
(z-\bar{z})^{2} \dot{\omega}_{\bar{z} z}^{\prime}[\alpha]\langle\bar{\delta}\rangle-2(z-\bar{z}) \dot{\omega}_{\bar{z}}^{\prime}[\alpha]\langle\bar{\delta}\rangle=(z-\bar{z})^{2}(\alpha \bar{\delta})_{\bar{z}} .
$$

But

$$
\begin{aligned}
(z-\bar{z})^{2} & \frac{\partial}{\partial \bar{z}}\left(-(z-\bar{z})^{2} \frac{\partial^{2}}{\partial z \partial \bar{z}}-2\right) \\
& =-\left((z-\bar{z})^{2} \frac{\partial}{\partial z}-2(z-\bar{z})\right)\left(\frac{\partial}{\partial \bar{z}}(z-\bar{z})^{2} \frac{\partial}{\partial \bar{z}}\right),
\end{aligned}
$$

and $\Delta=-(z-\bar{z})^{2}\left(\partial^{2} / \partial z \partial \bar{z}\right)$ is the Laplace-Beltrami operator so that we can write $(2.24)$ as

$$
\begin{aligned}
& (z-\bar{z})^{2} \dot{\omega}_{\bar{z} z}^{\prime}[\alpha]\langle\bar{\delta}\rangle-2(z-\bar{z}) \dot{\omega}_{\bar{z}}^{\prime}[\alpha]\langle\bar{\delta}\rangle \\
& =-\left((z-\bar{z})^{2} \frac{\partial}{\partial z}-2(z-\bar{z})\right)\left(\frac{\partial}{\partial \bar{z}}(z-\bar{z})^{2} \frac{\partial}{\partial \bar{z}}\left((\Delta-2)^{-1}(-\alpha \bar{\delta})\right)\right) .
\end{aligned}
$$

Now $(z-\bar{z})^{2}(\partial / \partial \bar{z})(\Delta-2)^{-1}(-\alpha \bar{\delta})$ again transforms as a vector field, namely with factor $\chi_{z}$, because $\alpha \bar{\delta}$ transforms as a function and $\Delta$ and hence $(\Delta-2)^{-1}$ is a zero order operator. Therefore,

$$
\frac{\partial}{\partial \bar{z}}\left((z-\bar{z})^{2} \frac{\partial}{\partial \bar{z}}\left((\Delta-2)^{-1}(-\alpha \bar{\delta})\right)\right)
$$

again is $L^{2}$-orthogonal to the harmonic Beltrami differentials.

Since, as remarked above, $\dot{\omega}_{\bar{z}}^{\prime}[\alpha]\langle\bar{\delta}\rangle$ is also orthogonal to the harmonic Beltrami differentials, we finally conclude

$$
\dot{\omega}_{\bar{z}}^{\prime}[\alpha]\langle\bar{\delta}\rangle=-\frac{\partial}{\partial \bar{z}}\left((z-\bar{z})^{2} \frac{\partial}{\partial \bar{z}}(\Delta-2)^{-1}(-\alpha \bar{\delta})\right) .
$$




\subsection{First derivatives of the metric. Kähler property}

In order to compute derivatives of $h_{\alpha \bar{\beta}}(\mu)$ at $\mu=0$, we pull formula (2.4) back to the base surface 0 , as in 1.2 :

$$
\begin{aligned}
h_{\alpha \bar{\beta}}(\mu)= & \int \frac{d^{2}}{d \alpha d \bar{\beta}}\left(\varrho^{2}(\omega) \omega_{z} \bar{\omega}_{\bar{z}} \zeta_{\bar{z}} \bar{\zeta}_{z}+\varrho^{2}(\omega) \omega_{\bar{z}} \bar{\omega}_{z} \zeta_{z} \bar{\zeta}_{\bar{z}}\right. \\
& -\varrho^{2}(\omega) \omega_{z} \bar{\omega}_{z} \bar{\zeta}_{\bar{z}} \zeta_{\bar{z}}-\varrho^{2}(\omega) \bar{\omega}_{\bar{z}} \omega_{\bar{z}} \zeta_{z} \bar{\zeta}_{z} \frac{1}{\zeta_{z} \bar{\zeta}_{\bar{z}}-\bar{\zeta}_{z} \zeta_{\bar{z}}} d z d \bar{z}
\end{aligned}
$$

Since at $\mu=0, \zeta(z)=z$, and

$$
\left(\frac{\zeta_{z} \bar{\zeta}_{\bar{z}}}{\zeta_{z} \bar{\zeta}_{\bar{z}}-\zeta_{\bar{z}} \bar{\zeta}_{z}}\right)^{\prime}[\gamma]=\left(\frac{\zeta_{\bar{z}} \bar{\zeta}_{z}}{\zeta_{z} \bar{\zeta}_{\bar{z}}-\zeta_{\bar{z}} \bar{\zeta}_{z}}\right)^{\prime}[\gamma]=0
$$

we compute, using (2.14) and (2.15),

$$
\begin{aligned}
h_{\alpha \bar{\beta}, \gamma}(0) & =\frac{d}{d \gamma} h_{\alpha \bar{\beta}}(0) \\
& =\int\left(\dot{\omega}_{\bar{z}}^{\prime}[\alpha]\langle\gamma\rangle \bar{\omega}_{z}^{\prime}[\beta]+\omega_{\bar{z}}^{\prime}[\alpha] \dot{\bar{\omega}}_{z}^{\prime}[\beta]\langle\gamma\rangle\right) \lambda^{2} d z d \bar{z}=0,
\end{aligned}
$$

as $\dot{\omega}^{\prime}$ transforms as an infinitesimal diffeomorphism (cf. (2.19)), and $\dot{\omega}_{\bar{z}}^{\prime}$ is hence orthogonal to the harmonic Beltrami differentials (cf. (2.7)).

Similarly,

$$
h_{\alpha \bar{\beta}, \bar{\delta}}(0)=0 \text {. }
$$

This yields the following result of Ahlfors and Weil, cf. [A1].

Theorem 1. The Weil-Petersson metric is a Kähler metric.

Namely, we have found normal coordinates which are holomorphic at our given point (but not elsewhere, actually), and this implies the Kähler property. In this regards, cf. also [Wp2].

Remark. The complex structure of Teichmüller space in terms of harmonic maps is discussed in [J2]. 


\subsection{Second derivatives. The curvature}

For the second derivatives, we let, as in 1.1

$$
H=\frac{\varrho^{2}(u)}{\lambda^{2}(z)} u_{z} \bar{u}_{\bar{z}}, \quad L=\frac{\varrho^{2}(u)}{\lambda^{2}(z)} u_{\bar{z}} \bar{u}_{z}
$$

for a harmonic map $u$, and denote complex derivatives at $\mu=0$ by corresponding subscripts: $H^{\prime \prime}[\alpha, \bar{\beta}](0)=H_{\alpha \bar{\beta}}$, etc.

The strategy is as follows:

We use (2.9), pulled back to the surface corresponding to the origin 0 by a change of variables, to write $h_{\alpha \bar{\beta}}(\mu)$ in two different forms. We differentiate both expressions and compare the results. Using (2.27) then yields an expression involving certain derivatives of $H$ and $L$. These have already been evaluated in 1.1, and the desired formula for the second derivatives of $h_{\alpha \bar{\beta}}$ will follow (cf. (2.38), (2.40)).

We compute first, using

$$
\left(\frac{\zeta_{z} \bar{\zeta}_{\bar{z}}}{\zeta_{z} \bar{\zeta}_{\bar{z}}-\zeta_{\bar{z}} \bar{\zeta}_{z}}\right)^{\prime \prime}[\gamma, \bar{\delta}]=\left(\frac{\zeta_{\bar{z}} \bar{\zeta}_{z}}{\zeta_{z} \bar{\zeta}_{\bar{z}}-\zeta_{\bar{z}} \bar{\zeta}_{z}}\right)^{\prime \prime}[\gamma, \bar{\delta}]=\gamma \bar{\delta},
$$

and (2.20), (2.22), (2.23) from (2.28)

$$
\begin{aligned}
h_{\alpha \bar{\beta}, \gamma \bar{\delta}}(0) & =\frac{d^{2}}{d \gamma d \bar{\delta}} h_{\alpha \bar{\beta}}(0)=\int\left(L_{\alpha \bar{\beta} \gamma \bar{\delta}}+H_{\alpha \bar{\beta}} \gamma \bar{\delta}+L_{\alpha \bar{\beta}} \gamma \bar{\delta}\right. \\
& +\ddot{\omega}_{\bar{z}}^{\prime}[\alpha]\langle\gamma, \bar{\delta}\rangle \bar{\beta}+\dot{\omega}_{\bar{z}}^{\prime}[\alpha]\left\langle\bar{\delta} \dot{\bar{\omega}}_{z}^{\prime}[\bar{\beta}]\langle\gamma\rangle+\alpha \ddot{\bar{\omega}}_{z}^{\prime}[\bar{\beta}]\langle\gamma, \bar{\delta}\rangle\right) \lambda^{2}(z) d z d \bar{z} .
\end{aligned}
$$

On the other hand, pulling (2.9) back to the surface 0 by a change of variables, we also have

$$
\begin{aligned}
h_{\alpha \bar{\beta}}=\int \frac{d^{2}}{d \alpha d \bar{\beta}} & \left(\varrho^{2}(\omega) \omega_{z} \bar{u}_{\bar{z}} \zeta_{\bar{z}} \bar{\zeta}_{z}+\varrho^{2}(\omega) \omega_{\bar{z}} \bar{u}_{z} \zeta_{z} \bar{\zeta}_{\bar{z}}\right. \\
& \left.-\varrho^{2}(\omega) \omega_{z} \bar{u}_{z} \zeta_{\bar{z}} \bar{\zeta}_{\bar{z}}-\varrho^{2}(\omega) \omega_{\bar{z}} \bar{u}_{\bar{z}} \zeta_{z} \zeta_{\bar{z}}\right) \frac{1}{\zeta_{z} \bar{\zeta}_{\bar{z}}-\zeta_{\bar{z}} \bar{\zeta}_{z}} d z d \bar{z}
\end{aligned}
$$

where, as before, $u: 0 \rightarrow \mu+\nu$ is harmonic.

Differentiating (2.34), we get

$$
h_{\alpha \bar{\beta}, \gamma \bar{\delta}}(0)=\int\left(L_{\alpha \bar{\beta} \gamma \bar{\delta}}+H_{\alpha \bar{\beta}} \gamma \bar{\delta}+L_{\alpha \bar{\beta}} \gamma \bar{\delta}+\ddot{\omega}_{\bar{z}}^{\prime}[\alpha]\langle\gamma, \bar{\delta}\rangle \bar{\beta}\right) \lambda^{2}(z) d z d \bar{z} .
$$

Comparing (2.33) and (2.35) yields

$$
\int\left(\dot{\omega}_{\bar{z}}^{\prime}[\alpha]\langle\bar{\delta}\rangle \dot{\bar{\omega}}_{z}^{\prime}[\bar{\beta}]\langle\gamma\rangle+\alpha \ddot{\bar{\omega}}_{z}^{\prime}[\bar{\beta}]\langle\gamma, \bar{\delta}\rangle\right) \lambda^{2}(z) d z d \bar{z}=0
$$


hence also

$$
\int \ddot{\omega}_{\bar{z}}^{\prime}[\alpha]\langle\gamma, \bar{\delta}\rangle \bar{\beta} \lambda^{2}(z) d z d \bar{z}=-\int \dot{\omega}_{\bar{z}}^{\prime}[\alpha]\left\langle\bar{\delta}_{\overline{\hat{\omega}}}^{\prime}[\bar{\beta}]\langle\gamma\rangle \lambda^{2}(z) d z d \bar{z}\right.
$$

and inserting this into (2.35) and using (2.27), we obtain

$$
\begin{aligned}
h_{\alpha \bar{\beta}, \gamma \bar{\delta}}(0)= & \int\left(L_{\alpha \bar{\beta} \gamma \bar{\delta}}+H_{\alpha \bar{\beta}} \gamma \bar{\delta}+L_{\alpha \bar{\beta}} \gamma \bar{\delta}\right. \\
& -\frac{\partial}{\partial \bar{z}}\left((z-\bar{z})^{2} \frac{\partial}{\partial \bar{z}}(\Delta-2)^{-1}(\alpha \bar{\delta})\right) \frac{\partial}{\partial z}\left((z-\bar{z})^{2} .\right. \\
& \left.\left.\cdot \frac{\partial}{\partial z}(\Delta-2)^{-1}(\bar{\beta} \gamma)\right)\right) \lambda^{2} d z d \bar{z} .
\end{aligned}
$$

In order to evaluate (2.37), we recall from Section 1.1

$$
\begin{aligned}
L_{\alpha \bar{\beta}} & =\alpha \bar{\beta} \\
H_{\alpha \bar{\beta}} & =-2(\Delta-2)^{-1}(\alpha \bar{\beta}) \\
L_{\alpha \bar{\beta} \gamma \bar{\delta}} & =-H_{\alpha \bar{\beta}} L_{\gamma \bar{\delta}}-H_{\gamma \bar{\delta}} L_{\alpha \bar{\beta}}-H_{\alpha \bar{\delta}} L_{\gamma \bar{\beta}}-H_{\gamma \bar{\beta}} L_{\alpha \bar{\delta}} .
\end{aligned}
$$

Also, when integrating the last term in (2.37) by parts, we obtain, since $\lambda^{2}(z)=$ $-2(z-\bar{z})^{-2}$, the operator

$$
\begin{aligned}
-\frac{\partial}{\partial \bar{z}} & \left((z-\bar{z})^{2} \frac{\partial}{\partial \bar{z}}\left(-\frac{2}{(z-\bar{z})^{2}} \frac{\partial}{\partial z}\left((z-\bar{z})^{2} \frac{\partial}{\partial z}\right)\right)\right) \\
& =2(z-\bar{z})^{2} \frac{\partial^{4}}{\partial z^{2} \partial \bar{z}^{2}}-4(z-\bar{z}) \frac{\partial^{3}}{\partial z^{2} \partial \bar{z}}+4(z-\bar{z}) \frac{\partial^{3}}{\partial \bar{z}^{2} \partial z} \\
& =\frac{2}{(z-\bar{z})^{2}}(\Delta \circ \Delta-2 \Delta), \text { since } \Delta=-(z-\bar{z})^{2} \frac{\partial^{2}}{\partial z \partial \bar{z}} \\
& =-\lambda^{2}(z)\left((\Delta-2)^{2}+2(\Delta-2)\right) .
\end{aligned}
$$

Since $(\Delta-2)$ is selfadjoint, we obtain altogether

$$
h_{\alpha \bar{\beta}, \gamma \bar{\delta}}(0)=\int\left\{\left(2(\Delta-2)^{-1}(\gamma \bar{\delta})\right) \alpha \bar{\beta}+\left(2(\Delta-2)^{-1}(\gamma \bar{\beta})\right) \alpha \bar{\delta}\right\} \lambda^{2}(z) d z d \bar{z}
$$

and also

$$
h_{\alpha \bar{\delta}, \gamma \bar{\beta}}(0)=h_{\alpha \bar{\beta}, \gamma \bar{\delta}}(0) .
$$

Since $H_{\alpha \gamma}=0=L_{\alpha \gamma}$, etc., $L_{\alpha \gamma \bar{\beta} \bar{\delta}}=L_{\alpha \bar{\beta} \gamma \bar{\delta}}$, we obtain likewise

$$
\begin{aligned}
h_{\alpha \gamma, \bar{\beta} \bar{\delta}}(0) & =\int L_{\alpha \bar{\beta} \gamma \bar{\delta}} \lambda^{2}(z) d z d \bar{z} \\
= & \int\left\{\left(4(\Delta-2)^{-1}(\gamma \bar{\delta})\right) \alpha \bar{\beta}+\left(4(\Delta-2)^{-1}(\gamma \bar{\beta})\right) \alpha \bar{\delta}\right\} \lambda^{2}(z) d z d \bar{z},
\end{aligned}
$$


and

$$
h_{\bar{\beta} \bar{\delta}, \alpha \gamma}(0)=h_{\alpha \gamma, \bar{\beta} \bar{\delta}} .
$$

This enables us to evaluate the curvature tensor $R_{\alpha \bar{\beta} \gamma \bar{\delta}}$ of the Weil-Petersson metric. The curvature of the Weil-Petersson metric has been computed by Tromba ([T1]), Wolpert (cf. [Wp] where such results are also independently attributed to Royden), and Siu ([Si]), by different methods.

Since, by (2.30), (2.31), first derivatives of the metric vanish, we have

$$
R_{\alpha \bar{\beta} \gamma \bar{\delta}}=\frac{1}{2}\left(h_{\alpha \bar{\beta}, \gamma \bar{\delta}}(0)+h_{\gamma \bar{\delta}, \alpha \bar{\beta}}(0)-h_{\alpha \gamma, \bar{\beta} \bar{\delta}}(0)-h_{\bar{\beta} \bar{\delta}, \alpha \gamma}(0)\right) .
$$

(Note that the quantities $h_{\alpha \gamma, \bar{\beta} \bar{\delta}}$ and $h_{\bar{\beta} \bar{\delta}, \alpha \gamma}$ appear, because our coordinate system is not holomorphic.)

From (2.38)-(2.42)

$$
R_{\alpha \bar{\beta} \gamma \bar{\delta}}=\int\left\{\left(-2(\Delta-2)^{-1}(\gamma \bar{\delta})\right) \alpha \bar{\beta}+\left(-2(\Delta-2)^{-1}(\gamma \bar{\beta})\right) \alpha \bar{\delta}\right\} \lambda^{2}(z) d z d \bar{z}
$$

From this formula, it is not hard to show that the sectional curvature of the Weil-Petersson metric is negative and that the holomorphic sectional and hence also the Ricci curvature are bounded from above by $-(2 \pi(p-1))^{-1}$. See [T1], [Wp], or [J2] for details.

\section{The geometry of the Teichmüller curve \\ 3.1. The metric of the vertical line bundle}

The Teichmüller curve $\mathcal{T}_{p}$ is a fibre bundle $\pi: \mathcal{T}_{p} \rightarrow T_{p}$ over Teichmüller space $T_{p}$ of surfaces of genus $p$, the fibre over $x \in T_{p}$ being the marked surface of genus $p$ represented by $x$. The universal cover of $\mathcal{T}_{p}$ is the Bers fibre space $\mathcal{B F}_{p}$, with a quasi-halfplane as fibre. The group $\bar{\Gamma}$ of covering transformations is isomorphic to the fundamental group of a surface of genus $p . \mathcal{B F}_{p}$ and the action of the fundamental group can be described as follows. For a surface $H / \Gamma=(\Sigma, g)$ representing $x \in T_{p}$, and $\psi \in Q(g)$, we put as before

$$
\mu(z):=\bar{\psi}(z) y^{2} .
$$

We assume $|\mu|_{L^{\infty}}<1$. We let $w^{\mu}$ be the homeomorphism $\mathbf{C} \rightarrow \mathbf{C}$ leaving 0,1, $\infty$ fixed and satisfying

$$
w_{\bar{z}}=\mu w_{z} \quad \text { in } H, \quad w_{\bar{z}}=0 \quad \text { in } \mathbf{C} \backslash H .
$$

We put

$$
\dot{\omega}[\alpha]:=\frac{d}{d t} w_{\mid t=0}^{t \alpha}
$$


Since, for $\gamma \in \Gamma,(\mu \circ \gamma) \bar{\gamma}_{\bar{z}}=\mu \gamma_{z}, w^{\mu} \circ \gamma$ also solves (3.1), for any $\gamma \in \Gamma$; hence there exists a group $\Gamma^{\mu}$ with $w^{\mu} \circ \gamma=\gamma_{\mu} \circ w^{\mu}$, for some $\gamma_{\mu} \in \Gamma^{\mu}$.

We fix a neighborhood $U$ of the origin in $\mathcal{H}(g)$ such that for $\mu \in U|\mu|_{L^{\infty}}<1$. Then, by a theorem of Bers [B], the map

$$
F: U \times H \rightarrow \mathcal{B F}_{p},(\mu, z) \rightarrow\left(\mu, w^{\mu}(z)\right)
$$

is holomorphic in $\mu$ (but not in $z$ ).

Then $\Gamma$ acts on $U \times H$ by $(\mu, z) \rightarrow(\mu, \gamma(z))$ and $\bar{\Gamma}$ acts on $\mathcal{B F}_{p}$ by $(\mu, w) \rightarrow$ $\left(\mu, \gamma^{\mu}(w)\right)$ and

$$
F(\mu, \gamma(z))=\left(\mu, \gamma^{\mu}\left(w^{\mu}(z)\right)\right)
$$

i.e. the local trivialization $F$ is $\Gamma-\bar{\Gamma}$ equivariant.

As $F$ is holomorphic in $\mu, \mu \rightarrow\left(\mu, w^{\mu}(z)\right)$, is, for fixed $z$, a holomorphic section of $\mathcal{B F}_{p}$ which, because of the equivariance of $F$, descends to a holomorphic section of $\mathcal{T}_{p}$. At $\mu=0$, a holomorphic tangent vector to this section is

$$
\tau(\alpha, z):=\dot{w}[\alpha] \frac{\partial}{\partial z}+\frac{\partial}{\partial \alpha} .
$$

This means that for a function $g(\mu, w(z, \mu))$, we have

$$
\tau(\alpha, z) g=\dot{w}[\alpha] \frac{\partial g}{\partial w}+\frac{\partial g}{\partial \alpha} .
$$

The vertical bundle of $\pi: \mathcal{T}_{p} \rightarrow T_{p}$ defines a line bundle $L$, obtained as $\operatorname{Ker}(d \pi)$, where

$$
d \pi: T^{1,0} \mathcal{T}_{p} \rightarrow T^{1,0} T_{p}
$$

The restriction of $L$ to a fibre is isomorphic to the tangent bundle of this fibre. Furthermore, because $F$ is holomorphic in $\mu, \partial / \partial w$ provides a local holomorphic section of $L$.

For $\mu \in U$, we let $f^{\mu}$ be the homeomorphism solution of

$$
f^{\mu}: H \rightarrow H, \quad f_{\bar{z}}^{\mu}=\mu f_{z}^{\mu},
$$

fixing $0,1, \infty$.

Thus, there exists a conformal map $g: w^{\mu}(H) \rightarrow H$ with $f^{\mu}=g \circ w^{\mu}$. We denote the hyperbolic metric in $H$ by $\varrho^{2}(f) d f d \bar{f}$ and in $w^{\mu}(H)$ by $\sigma^{2}(w) d w d \bar{w}$. Then

$$
\left\|\frac{\partial}{\partial w}\right\|^{2}=\sigma^{2}(w)=\varrho^{2}(g(w)) \frac{\left|f_{z}^{\mu}\right|^{2}}{\left|w_{z}^{\mu}\right|^{2}} .
$$


Now,

$$
\begin{aligned}
\frac{1}{2} i \varrho^{2}(g(w))\left|f_{z}^{\mu}\right|^{2} & \left(1-|\mu|^{2}\right) d z \\
= & \wedge d \bar{z} \\
& i \varrho^{2}(g(w))\left(\left|f_{z}^{\mu}\right|^{2}-\left|f_{\bar{z}}^{\mu}\right|^{2}\right) d z \wedge d \bar{z}=\frac{1}{2} i \varrho^{2}(g(w)) d f^{\mu} \wedge \overline{d f^{\mu}}
\end{aligned}
$$

is the hyperbolic area element.

We write

$$
J(\mu):=\varrho^{2}(g(w))\left|f_{z}^{\mu}\right|^{2}\left(1-|\mu|^{2}\right) .
$$

Then

$$
\left\|\frac{\partial}{\partial w}\right\|^{2}=\frac{J(\mu)}{\left|w_{z}^{\mu}\right|^{2}\left(1-|\mu|^{2}\right)} .
$$

We now compute the connection $\Theta$ and curvature $\Omega$ of the induced metric on $L$ from the above formula for the length of the holomorphic section $\partial / \partial w$, following Wolpert [Wp].

We shall evaluate all expressions at the origin of $\mathcal{H}(g)$. Then $\alpha$ is a tangent vector at the origin. Observing that at the origin $w^{0}(z)=z$, hence, at the origin

$$
\left\|\frac{\partial}{\partial w}\right\|=\frac{-4}{(z-\bar{z})^{2}}
$$

we have, for the connection 1 -form

$$
\Theta=\partial \log \left\|\frac{\partial}{\partial w}\right\|^{2}
$$

at the origin

$$
\Theta\left(\frac{\partial}{\partial z}\right)=-\frac{2}{(z-\bar{z})}
$$

In order to compute $\Theta\left(\tau_{\alpha}\right)$, writing $\tau_{\alpha}:=\tau(\alpha, \cdot)$, we note at the origin

$$
\frac{\partial}{\partial \tau_{\alpha}} J(\mu)=0
$$

(cf. (1.29)), and, since $w^{\mu}(z)$ is holomorphic in $\mu$, we obtain at the origin

$$
\Theta\left(\tau_{\alpha}\right)=-\dot{w}[\alpha]_{z}
$$


We proceed to compute the curvature 2 -form

$$
\Omega=\bar{\partial} \partial \log \left\|\frac{\partial}{\partial w}\right\|^{2}
$$

First of all, as before at the origin

$$
\Omega\left(\frac{\partial}{\partial \bar{z}}, \frac{\partial}{\partial z}\right)=-\frac{2}{(z-\bar{z})^{2}} .
$$

Next

$$
\begin{aligned}
\Omega\left(\frac{\partial}{\partial \bar{z}}, \tau_{\alpha}\right) & =\frac{d}{d \tau_{\alpha}} \frac{\partial}{\partial \bar{z}} \log \left\|\frac{\partial}{\partial w}\right\|^{2} \\
& =\frac{\partial}{\partial \bar{z}} \frac{d}{d \tau_{\alpha}} \log \left\|\frac{\partial}{\partial w}\right\|^{2}-\frac{\partial}{\partial z}\left(\log \left\|\frac{\partial}{\partial w}\right\|^{2}\right) \dot{w}[\alpha]_{\bar{z}} \\
& =-\dot{w}[\alpha]_{z \bar{z}}+\frac{2}{z-\bar{z}} \dot{w}[\alpha]_{\bar{z}} .
\end{aligned}
$$

From (3.1), at the origin

$$
\dot{w}[\alpha]_{\bar{z}}=\alpha .
$$

Since $\alpha$ is a harmonic Beltrami differential, i.e. $\alpha=-\frac{1}{4}(z-\bar{z})^{2} \bar{\psi}$, with holomorphic $\psi$,

$$
\alpha_{z}-\frac{2}{z-\bar{z}} \alpha=0
$$

and thus

$$
\Omega\left(\frac{\partial}{\partial \bar{z}}, \tau_{\alpha}\right)=0
$$

Finally,

$$
\Omega\left(\bar{\tau}_{\beta}, \tau_{\alpha}\right)=\frac{d^{2}}{d \bar{\tau}_{\beta} d \tau_{\alpha}} \log \left\|\frac{\partial}{\partial w}\right\|^{2}
$$

Recalling (3.6), we note (always at the origin)

$$
\frac{d^{2}}{d \bar{\tau}_{\beta} d \tau_{\alpha}} \log J(\mu)=D(\alpha \bar{\beta})-\alpha \bar{\beta},
$$

with

$$
D(\alpha \bar{\beta}):=-2(\Delta-2)^{-1}(\alpha \bar{\beta}),
$$


cf. (1.29), (1.30), (1.31),

$$
-\frac{d^{2}}{d \bar{\tau}_{\beta} d \tau_{\alpha}} \log \left|w_{z}^{\mu}\right|^{2}=0
$$

since $w^{\mu}$ is holomorphic in $\mu$,

$$
-\frac{d^{2}}{d \bar{\tau}_{\beta} d \tau_{\alpha}} \log \left(1-|\mu|^{2}\right)=\alpha \bar{\beta}
$$

hence altogether

$$
\Omega\left(\bar{\tau}_{\beta}, \tau_{\alpha}\right)=D(\alpha \bar{\beta})
$$

\subsection{A metric on the Teichmüller curve and its properties}

As a consequence of (3.10), (3.13), (3.18), Wolpert [Wp] observed that the first Chern class $c_{1}(L)=-\Omega / 2 \pi i$ is negative. Moreover, we obtain a Kähler metric on $\mathcal{B F}_{p}$ and $\mathcal{T}_{p}$ by using $-c_{1}(L)$ as Kähler form.

We now want to compute the curvature tensor of this Kähler metric. In order to fix the notation, we may suppose that the point under consideration is in the fibre over $0 \in U$.

We select a basis $\alpha, \beta, \gamma, \ldots$ of $\mathcal{H}(g)$ and consider $\tau_{\alpha}, \tau_{\beta}, \ldots$ as holomorphic tangent vectors at the point under consideration. We then have as metric tensor, at the considered point $\left(0, z_{0}\right)$,

$$
\begin{gathered}
g_{1 \overline{1}}=\Omega\left(\frac{\partial}{\partial \bar{z}}, \frac{\partial}{\partial z}\right)=\frac{-2}{(z-\bar{z})^{2}}, \\
g_{\alpha \overline{1}}=\Omega\left(\frac{\partial}{\partial \bar{z}}, \tau_{\alpha}\right)=0 \\
g_{\alpha \bar{\beta}}=\Omega\left(\bar{\tau}_{\beta}, \tau_{\beta}\right)=D(\alpha \bar{\beta}) .
\end{gathered}
$$

We note that as a consequence of the maximum principle, $D(\mu \bar{\nu})\left(z_{0}\right)$ is a positive definite quadratic form. Therefore, we can choose $\alpha, \beta, \gamma, \ldots$ in such a way that

$$
D(\mu \bar{\nu})\left(z_{0}\right)=\delta_{\mu \nu} \quad(=1 \text { if } \mu=\nu,=0 \text { otherwise }),
$$

for all elements of our basis. 
Harmonic maps and curvature computations in Teichmüller theory

For simplicity of notation, we put

$$
h(\mu, w(z, \mu)):=\log \left\|\frac{\partial}{\partial w}\right\|^{2} .
$$

Furthermore, a subscript will always denote a partial derivative. Thus

$$
\begin{gathered}
\frac{d}{d \tau_{\alpha}} h=h_{\alpha}+h_{w} \dot{w}[\alpha], \\
\frac{d}{d \bar{\tau}_{\beta}} h=h_{\beta}+h_{\bar{w}} \overline{\dot{w}}[\beta],
\end{gathered}
$$

since $w$ depends holomorphically on $\mu$.

We saw already in the derivation of (3.13) that the vector fields $\tau_{\alpha}$ and $\partial / \partial \bar{z}$ do not commute. As a consequence, also in our subsequent expressions, we always have to subtract certain commutator terms.

We note, for $g=g(\mu, w(z, \mu))$,

$$
\frac{d}{d \tau_{\alpha}} \frac{\partial}{\partial \bar{z}} g=\frac{\partial}{\partial \bar{z}} \frac{d}{d \tau_{\alpha}} g-g_{w} \dot{w}[\alpha]_{\bar{z}}
$$

$$
\frac{d}{d \bar{\tau}_{\beta}} \frac{\partial}{\partial z} g=\frac{\partial}{\partial z} \frac{d}{d \bar{\tau}_{\beta}} g-g_{\bar{w}} \overline{\dot{w}}[\beta]_{z}
$$

$$
\frac{d}{d \tau_{\alpha}} \frac{\partial}{\partial z} g=\frac{\partial}{\partial z} \frac{d}{d \tau_{\alpha}} g-g_{w} \dot{w}[\alpha]_{z}
$$

$$
\frac{d}{d \bar{\tau}_{\beta}} \frac{\partial}{\partial \bar{z}} g=\frac{\partial}{\partial \bar{z}} \frac{d}{d \bar{\tau}_{\beta}} g-g_{\bar{w}} \overline{\dot{w}}[\beta]_{\bar{z}} .
$$

Whenever the variable $z$ occurs, it always refers to evaluation at the origin $\mu=0$. For reference, we also note

$$
\frac{\partial}{\partial z} h=\frac{\partial}{\partial z} \log \frac{-4}{(z-\bar{z})^{2}}=\frac{-2}{(z-\bar{z})},
$$

$$
\frac{\partial}{\partial \bar{z}} h=\frac{2}{z-\bar{z}}
$$




$$
g_{1 \overline{1}}=\frac{\partial^{2}}{\partial z \partial \bar{z}} h=\frac{-2}{(z-\bar{z})^{2}}
$$

$$
\frac{\partial^{2}}{\partial z^{2}} h=\frac{\partial^{2}}{\partial \bar{z}^{2}} h=\frac{2}{(z-\bar{z})^{2}}
$$

$$
g_{1 \overline{1}, 1}=\frac{\partial}{\partial z} \frac{-2}{(z-\bar{z})^{2}}=\frac{4}{(z-\bar{z})^{3}}
$$

$$
g_{1 \overline{1}, \overline{1}}=\frac{\partial}{\partial \bar{z}} \frac{-2}{(z-\bar{z})^{2}}=-\frac{4}{(z-\bar{z})^{3}}
$$

$$
g_{1 \overline{1}, 1 \overline{1}}=\frac{\partial^{2}}{\partial z \partial \bar{z}} \frac{-2}{(z-\bar{z})^{2}}=\frac{12}{(z-\bar{z})^{4}}
$$

$$
\frac{d}{d \tau_{\alpha}} h=-\dot{w}[\alpha]_{z}
$$

cf. (3.9),

$$
h_{\alpha}=\frac{d}{d \tau_{\alpha}} h-h_{z} \dot{w}[\alpha]=\frac{2}{z-\bar{z}} \dot{w}[\alpha]-\dot{w}[\alpha]_{z},
$$

cf. (3.23), (3.36), (3.29),

$$
h_{\alpha \bar{z}}=-\dot{w}[\alpha]_{z \bar{z}}+\frac{2}{(z-\bar{z})^{2}} \dot{w}[\alpha]+\frac{2}{z-\bar{z}} \dot{w}[\alpha]_{\bar{z}}=\frac{2}{(z-\bar{z})^{2}} \dot{w}[\alpha],
$$

cf. (3.25), (3.26), (3.31), (3.29), (3.11), (3.12),

$$
h_{\bar{\beta} z}=\frac{2}{(z-\bar{z})^{2}} \overline{\dot{w}}[\beta]
$$

$$
\frac{d}{d \tau_{\alpha}} h_{z}=-\dot{w}[\alpha]_{z z}+\frac{2}{z-\bar{z}} \dot{w}[\alpha]_{z}
$$

cf. (3.27), (3.36), (3.29),

$$
\frac{d}{d \tau_{\alpha}} h_{\bar{z}}=-\dot{w}[\alpha]_{z \bar{z}}+\frac{2}{z-\bar{z}} \dot{w}[\alpha]_{\bar{z}}=0
$$


cf. (3.13)

$$
\frac{d}{d \bar{\tau}_{\beta}} h_{z}=0
$$

$$
\frac{d}{d \bar{\tau}_{\beta}} h_{\bar{z}}=-\overline{\dot{w}}[\beta]_{\bar{z} \bar{z}}-\frac{2}{z-\bar{z}} \overline{\dot{w}}[\beta]_{\bar{z}}
$$

$$
h_{\alpha \bar{\beta}}=D(\alpha \bar{\beta}) \text {, }
$$

cf. (3.18),

$$
h_{\alpha z}=-\dot{w}[\alpha]_{z z}-\frac{2}{(z-\bar{z})^{2}} \dot{w}[\alpha]+\frac{2}{z-\bar{z}} \dot{w}[\alpha]_{z},
$$

cf. (3.27), (3.36), (3.32), (3.29),

$$
h_{\bar{\beta} \bar{z}}=-\overline{\dot{w}}[\beta]_{\bar{z} \bar{z}}-\frac{2}{(z-\bar{z})} \overline{\dot{w}}[\beta]-\frac{2}{z-\bar{z}} \overline{\dot{w}}[\beta]_{\bar{z}} .
$$

We proceed to compute

$$
g_{1 \overline{1}, \alpha}=\frac{d}{d \tau_{\alpha}} \frac{\partial^{2}}{\partial z \partial \bar{z}} h=\frac{\partial}{\partial \bar{z}} \frac{d}{d \tau_{\alpha}} h_{z}-\frac{\partial^{2}}{\partial z^{2}} h \cdot \dot{w}[\alpha]_{\bar{z}},
$$

by $(3.25)$

$$
\begin{gathered}
=-\dot{w}[\alpha]_{z z \bar{z}}+\frac{2}{(z-\bar{z})^{2}} \dot{w}[\alpha]_{z}+\frac{2}{z-\bar{z}} \dot{w}[\alpha]_{z \bar{z}}-\frac{2}{(z-\bar{z})^{2}} \dot{w}[\alpha]_{\bar{z}} \\
=\frac{2}{(z-\bar{z})^{2}} \dot{w}[\alpha]_{z}
\end{gathered}
$$

by (3.11), (3.12). Alternatively,

$$
g_{1 \overline{1}, \alpha}=\frac{d}{d \tau_{\alpha}}\left(\frac{1}{2} \frac{J(\mu)}{\left|w_{z}^{\mu}\right|^{2}\left(1-|\mu|^{2}\right)}\right)=\frac{2}{(z-\bar{z})^{2}} \dot{w}[\alpha]_{z},
$$

since $\left(d / d \tau_{\alpha}\right) J(\alpha)=0$, by (1.29).

Moreover,

$$
g_{1 \bar{\beta}, 1}=\frac{d}{d \bar{\tau}_{\beta}} \frac{\partial^{2}}{\partial z^{2}} h=\frac{\partial^{2}}{\partial z^{2}} \frac{d}{d \bar{\tau}_{\beta}} h-2 h_{\bar{z} z} \overline{\dot{w}}[\beta]_{z}-h_{\bar{z}} \overline{\dot{w}}[\beta]_{z z},
$$


by $(3.26)$

$$
=-\overline{\dot{w}}[\beta]_{\bar{z} z z}+\frac{4}{(z-\bar{z})^{2}} \overline{\dot{w}}[\beta]_{z}-\frac{2}{z-\bar{z}} \overline{\dot{w}}[\beta]_{z z},
$$

by $(3.36),(3.31),(3.30)$,

$$
=\frac{2}{(z-\bar{z})^{2}} \beta
$$

by using $\overline{\dot{w}}[\beta]_{z}=\bar{\beta}$ and differentiating the relation

$$
\bar{\beta}_{\bar{z}}+\frac{2}{z-\bar{z}} \bar{\beta}=0
$$

w.r.t. $z$.

$$
g_{1 \bar{\beta}, \alpha}=\frac{d^{2}}{d \bar{\tau}_{\beta} d \tau_{\alpha}} h_{z}=h_{z \alpha \bar{\beta}}+h_{z \alpha \bar{z}} \overline{\dot{w}}[\beta]+h_{z \bar{\beta} z} \dot{w}[\alpha]+h_{z z \bar{z}} \dot{w}[\alpha] \overline{\dot{w}}[\beta] .
$$

Since

$$
\begin{aligned}
\frac{\partial}{\partial z} \frac{d^{2}}{d \bar{\tau}_{\beta} d \tau_{\alpha}} h= & h_{z \alpha \bar{\beta}}+h_{z \bar{\beta} z} \dot{w}[\alpha]+h_{z \bar{\beta}} \dot{w}[\alpha]_{z}+h_{\alpha \bar{z} z} \overline{\dot{w}}[\beta]+h_{\alpha \bar{z}} \overline{\dot{w}}[\beta]_{z} \\
& +h_{z \bar{z}} \dot{w}[\alpha]_{z} \overline{\bar{w}}[\beta]+h_{z \bar{z}} \dot{w}[\alpha] \overline{\dot{w}}[\beta]_{z}+h_{z z \bar{z}} \dot{w}[\alpha] \bar{w}[\beta]
\end{aligned}
$$

we obtain, from (3.44), (3.38), (3.39), (3.31)

$$
\begin{aligned}
g_{1 \bar{\beta}, \alpha}= & \frac{\partial}{\partial z} D(\alpha \bar{\beta})-\frac{2}{(z-\bar{z})^{2}} \overline{\dot{w}}[\beta] \dot{w}[\alpha]_{z}-\frac{2}{(z-\bar{z})^{2}} \dot{w}[\alpha] \overline{\dot{w}}[\beta]_{z} \\
& +\frac{2}{(z-\bar{z})^{2}} \dot{w}[\alpha]_{z} \overline{\dot{w}}[\beta]+\frac{2}{(z-\bar{z})^{2}} \dot{w}[\alpha] \overline{\dot{w}}[\beta]_{z} \\
= & \frac{\partial}{\partial z} D(\alpha \bar{\beta})
\end{aligned}
$$

and likewise

$$
\begin{gathered}
g_{\overline{1} \bar{\beta}, \alpha}=\frac{\partial}{\partial \bar{z}} D(\alpha \bar{\beta}) . \\
g_{\alpha \overline{1}, \gamma}=\frac{d^{2}}{d \tau_{\alpha} d \tau_{\gamma}} h_{\bar{z}}=\frac{\partial}{\partial \bar{z}} \frac{d^{2}}{d \tau_{\alpha} d \tau_{\gamma}} h-\left(\frac{d}{d \tau_{\gamma}} h_{z}\right) \dot{w}[\alpha]_{\bar{z}} \\
-\left(\frac{d}{d \tau_{\alpha}} h_{z}\right) \dot{w}[\gamma]_{\bar{z}}-h_{z} \ddot{w}[\alpha, \gamma]_{\bar{z}}
\end{gathered}
$$


by (3.25). Now

$$
\frac{d^{2}}{d \tau_{\alpha} d \tau_{\gamma}} h=\frac{d^{2}}{d \tau_{\alpha} d \tau_{\gamma}} \log \frac{J(\mu)}{\left|w_{z}^{\mu}\right|^{2}\left(1-|\mu|^{2}\right)}=-\ddot{w}[\alpha, \gamma]_{z}+\dot{w}[\alpha]_{z} \dot{w}[\gamma]_{z}
$$

from (1.29), (1.32). Thus, using (3.40), (3.29)

$$
\begin{aligned}
g_{\alpha \overline{1}, \gamma}= & -\ddot{w}[\alpha, \gamma]_{\bar{z} z}+\dot{w}[\alpha]_{\bar{z} z} \dot{w}[\gamma]_{z}+\dot{w}[\alpha]_{z} \dot{w}[\gamma]_{\bar{z} z}+\dot{w}[\gamma]_{z z} \dot{w}[\alpha]_{\bar{z}} \\
& +\dot{w}[\alpha]_{z z} \dot{w}[\gamma]_{\bar{z}}-\frac{2}{z-\bar{z}} \dot{w}[\gamma]_{z} \dot{w}[\alpha]_{\bar{z}}-\frac{2}{z-\bar{z}} \dot{w}[\alpha]_{z} \dot{w}[\gamma]_{\bar{z}}+\frac{2}{z-\bar{z}} \ddot{w}[\alpha, \gamma]_{\bar{z}}
\end{aligned}
$$

Now, since $w_{\bar{z}}^{\mu}=\mu w_{z}$, we have $\dot{w}_{\bar{z}}^{\mu}[\alpha]=\alpha w_{z}^{\mu}+\mu \dot{w}_{z}^{\mu}[\alpha]$, hence, at $\mu=0$,

$$
\ddot{w}_{\bar{z}}[\alpha, \gamma]=\alpha \dot{w}[\gamma]_{z}+\gamma \dot{w}[\alpha]_{z},
$$

and of course,

$$
\dot{w}[\alpha]_{\bar{z}}=\alpha, \quad \dot{w}[\gamma]_{\bar{z}}=\gamma
$$

and one computes

$$
g_{\alpha \overline{1}, \gamma}=0
$$

Then

$$
g_{\alpha \bar{\beta}, \gamma}=\frac{d^{3}}{d \tau_{\gamma} d \bar{\tau}_{\beta} d \tau_{\alpha}} h=\frac{d^{3}}{d \tau_{\gamma} d \bar{\tau}_{\beta} d \tau_{\alpha}} \log J(\mu)
$$

from (3.6), since $w^{\mu}$ is holomorphic in $\mu$. Thus

$$
g_{\alpha \bar{\beta}, \gamma}=\frac{d}{d \tau_{\gamma}} D(\alpha \bar{\beta})
$$

by (3.44).

We now draw some consequences of the preceding formulae.

First recall that for each surface $(\Sigma, g)=H / \Gamma$,

$$
\mu \rightarrow\left(\mu, w^{\mu}(z)\right) \text { for } \quad \mu \in \mathcal{H}(g)
$$

yields a holomorphic section of $\mathcal{B F}_{p}$ and $\mathcal{T}_{p}$. At $\mu=0$, which corresponds to the surface $(\Sigma, g)$ itself, holomorphic tangent vectors are given by

$$
\tau(\alpha, z)=\dot{w}[\alpha] \frac{\partial}{\partial z}+\frac{\partial}{\partial \alpha} \quad(\alpha \in \mathcal{H}(g))
$$


cf. (3.3). We can construct an envelope of these sections with a holomorphic tangent plane at each $(\Sigma, g)=H / \Gamma$ spanned by the vectors $\tau(\alpha, z)$. Namely, the vectors $\tau(\alpha, z)$ and $\tau(\gamma, z)$ or $\tau(\bar{\beta}, z)$ commute; e.g.

$$
\begin{aligned}
\frac{d}{d \tau_{\gamma}} \frac{d}{d \tau_{\alpha}} g(\mu, w(z, \mu)) & =\left(\dot{w}[\gamma] \frac{\partial}{\partial w}+\frac{\partial}{\partial \gamma}\right)\left(\dot{w}[\alpha] g_{w}+g_{\alpha}\right) \\
& =\ddot{w}[\alpha, \gamma] g_{w}+\dot{w}[\gamma] \dot{w}[\alpha] g_{w w}+\dot{w}[\alpha] g_{w \gamma}+\dot{w}[\gamma] g_{\alpha w}+g_{\alpha \gamma},
\end{aligned}
$$

and this is symmetric in $\alpha$ and $\gamma$ (note that no $w$-derivative of $\dot{w}[\alpha]$ occurs as $\dot{w}[\alpha]$ is a function of the variable $z$ and not of $w$ ).

Likewise

$$
\frac{d}{d \bar{\tau}_{\beta}} \frac{d}{d \tau_{\alpha}} g=\dot{\bar{w}}[\bar{\beta}] \dot{w}[\alpha] g_{w \bar{w}}+\dot{w}[\alpha] g_{w \bar{\beta}}+\dot{\bar{w}}[\bar{\beta}] g_{\alpha \bar{w}}+g_{\alpha \bar{\beta}}
$$

since $w$ is holomorphic in $\mu$.

Consequently, the vectors $\tau(\alpha, \tau), \tau(\bar{\beta}, z)$ for $\alpha$ and $\beta$ varying holomorphically form an integrable distribution. The integral manifolds then are the desired envelopes.

Theorem 2. This enveloping section with tangent vectors $\tau(\alpha, z)$ in $\mathcal{B F}_{p}$ (or $\mathcal{T}_{p}$ ) is totally geodesic.

Proof. A submanifold $N$ of a Riemannian manifold $M$ is totally geodesic if any geodesic in $N$ (w.r.t. its intrinsic metric) is also a geodesic in $M$.

We use indices $i, j, k$ for $N$ and $p, q, r$ for $M$. Let $c=c(t)$ be a geodesic in $N$. Then, a dot denoting a derivative w.r.t. $t$,

$$
\ddot{c}^{i}+\Gamma_{j k}^{i} \dot{c}^{j} \dot{c}^{k}=0
$$

for all $i$. If $c$ is also geodesic in $M$, then

$$
\ddot{c}^{p}+\Gamma_{q r}^{p} \dot{c}^{q} \dot{c}^{r}=0
$$

for all $p$.

We choose the indices $p=1, \ldots, m(=\operatorname{dim} M)$ in such a way that the indices $1, \ldots, n(=\operatorname{dim} N)<m$ represent directions tangent to $N$, and the ones $n+1, \ldots, m$ directions transversal to $N$. If $c$ is geodesic in $M$, we then infer

$$
\Gamma_{q r}^{p} \dot{c}^{q} \dot{c}^{r}=0
$$

for $p=n+1, \ldots, m$. Returning to the present situation, we have to verify that the section $\mu \rightarrow\left(\mu, w^{\mu}(z)\right)$ is totally geodesic at $\mu=0$. Therefore, it suffices to show $\Gamma_{\alpha \beta}^{1}=0$ for all $\alpha, \beta$. We have

$$
\Gamma_{\alpha \beta}^{1}=\frac{1}{2} g^{1 \overline{1}}\left(g_{\alpha \overline{1}, \beta}+g_{\beta \overline{1}, \alpha}-g_{\alpha \beta, \overline{1}}\right)
$$

since the metric tensor is diagonal (at $\mu=0$ ), cf. (3.20).

Now $g_{\alpha \overline{1}, \beta}$ and $g_{\beta \overline{1}, \alpha}$ vanish by (3.55), and $g_{\alpha \beta, \overline{1}}$ vanishes since $g_{\alpha \beta}$ vanishes identically for $\mu=0$, as a consequence of (1.32) (one just has to repeat the arguments leading to (3.21)). This concludes the proof. QED.

Concerning the curvature, we have the following negative result: 
Theorem 3. $\mathcal{T}_{p}$ does not have nonpositive holomorphic sectional curvature in the directions of its fibers.

Proof. The general formula for the curvature tensor of a Kähler metric is

$$
R_{i \bar{j} k \bar{l}}=g_{i \bar{j}, k \bar{l}}-\sum_{s, t} g^{s \bar{t}} g_{i \bar{t}, k} g_{s \bar{j}, \bar{l}}
$$

Since the metric tensor is diagonal $((3.20)), g_{\alpha \bar{\alpha}}=1((3.21),(3.22))$, and $g_{1 \overline{1}}=$ $-2 /(z-\bar{z})^{2}((3.19))$, we obtain

$$
R_{1 \overline{1} 1 \overline{1}}=g_{1 \overline{1}, 1 \overline{1}}+\frac{1}{2}(z-\bar{z})^{2} g_{1 \overline{1}, 1} g_{1 \overline{1}, \overline{1}}-\sum_{\alpha} g_{1 \bar{\alpha}, 1} g_{\alpha \overline{1}, \overline{1}}=\frac{4}{(z-\bar{z})^{4}}\left(1-\sum_{\alpha} \alpha \bar{\alpha}\right)
$$

from (3.35), (3.34), (3.48).

The holomorphic sectional curvature in the fiber direction, i.e. the sectional curvature of the tangent plane spanned by $\partial / \partial z$ and $i \partial / \partial z$, is then given as

$$
-\left(1-\sum_{\alpha} \alpha \bar{\alpha}\right)\left(z_{0}\right)
$$

Since, at $z_{0}$, we have normalized the tangent vectors $\alpha$ in such a way that $D(\alpha \bar{\alpha})\left(z_{0}\right)=1$ for all basis vectors $\alpha$, cf. (3.22), we infer from the maximum principle that (3.59) cannot be nonpositive. QED.

\section{Bibliography}

[A1] Ahlfors, L.: Some remarks on Teichmüller's space of Riemann surfaces. - Ann. Math. 74, 1961, 171-191.

[A2] Ahlfors, L.: Curvature properties of Teichmüller's space. - J. Analyse Math. 9, 1961, 161-176.

[AB] Ahlfors, L., and L. Bers: Riemann's mapping theorem for variable metrics. - Ann. Math. 72, 1960, 385-404.

[Al1] AL'BER: On $n$-dimensional problems in the calculus of variations in the large. - Soviet Math. Dokl. 5, 1964, 700-704.

[Al2] AL'BER: Spaces of mappings into a manifold with negative curvature. - Soviet Math. Dokl. 9, 1967, 6-9.

[B] Bers, L.: Fibre spaces over Teichmüller spaces. - Acta Math. 130, 1973, 89-126.

[ES] Eells, J., and J. SAmpson: Harmonic mappings of Riemannian manifolds. - Amer. J. Math. 86, 1964, 109-160.

[H] Hartman, P.: On homotopic harmonic maps. - Canad. J. Math. 19, 1967, 673-687.

[J1] Jost, J.: Harmonic maps between surfaces. - Lecture Notes in Mathematics 1062, Springer-Verlag, 1984.

[J2] JosT, J.: Two dimensional geometric variational problems. - Wiley-Interscience, Chichester, 1991. 
[JY] JosT, J., and S.T. YAU: Harmonic maps and algebraic varieties over function fields. Preprint.

[Sa] SAmpson, J.H.: Some properties and applications of harmonic mappings. - Ann. Sci. École Norm. Sup. 11, 1978, 211-228.

[SY] Schoen, R., and S.T. YAU: On univalent harmonic maps between surfaces. - Invent. Math. 44, 1978, 265-278.

[Si] SiU, Y.T.: Curvature of the Weil-Petersson metric in the moduli space of compact KählerEinstein manifolds of negative first Chern class. - Aspects of Math. 9, 1986, 262-298.

[T1] Tromba, A.: On a natural algebraic affine connection on the space of almost complex structures and the curvature of Teichmüller space with respect to its Weil-Petersson metric. - Manuscripta Math. 56, 1986, 475-497.

[T2] Tromba, A.: On an energy function for the Weil-Petersson metric on Teichmüller space. - Manuscripta Math. 59, 1987, 249-260.

[Wf] Wolf, M.: The Teichmüller theory of harmonic maps. - J. Differential Geom. 29, 1989, 449-479.

[Wp1] Wolpert, S.: Chern forms and the Riemann tensor for the moduli space of curves. Invent. Math. 85, 1986, 119-145.

[Wp2] WolperT, S.: The Bers embedding and the Weil-Petersson metric. - Duke Math. J. 60, 1990, 497-508.

Ruhr-Universität Bochum

Fakultät für Mathematik

Universitätsstraße 150

D-4630 Bochum

Bundesrepublik Deutschland

Received 12 June 1989 